\title{
Blocking IFNAR1 inhibits multiple myeloma-driven Treg expansion and immunosuppression
}

\author{
Yawara Kawano,, ${ }^{1,2}$ Oksana Zavidij, ${ }^{1}$ Jihye Park, ${ }^{1}$ Michele Moschetta, ${ }^{1}$ Katsutoshi Kokubun, ${ }^{1}$ Tarek H. Mouhieddine, ${ }^{1}$ \\ Salomon Manier, ${ }^{1}$ Yuji Mishima, ${ }^{1}$ Naoka Murakami, ${ }^{3}$ Mark Bustoros, ${ }^{1}$ Romanos Sklavenitis Pistofidis, ${ }^{1}$ Mairead Reidy, ${ }^{1}$ \\ Yu J. Shen, ${ }^{1}$ Mahshid Rahmat, ${ }^{1}$ Pavlo Lukyanchykov, ${ }^{3}$ Esilida Sula Karreci, ${ }^{3}$ Shokichi Tsukamoto, ${ }^{1}$ Jiantao Shi, ${ }^{4}$ Satoshi Takagi, ${ }^{1}$ \\ Daisy Huynh, ${ }^{1}$ Antonio Sacco, ${ }^{1,5}$ Yu-Tzu Tai, ${ }^{1}$ Marta Chesi, ${ }^{6}$ P. Leif Bergsagel, ${ }^{6}$ Aldo M. Roccaro, ${ }^{1,5}$ Jamil Azzi, ${ }^{3}$ and Irene M. Chobrial' \\ 'Department of Medical Oncology, Dana-Farber Cancer Institute, Harvard Medical School, Boston, Massachusetts, USA. ²Department of Hematology, Kumamoto University Hospital, Kumamoto, Japan. \\ ${ }^{3}$ Transplantation Research Center, Renal Division, Brigham and Women's Hospital, and ${ }^{4}$ Department of Biostatistics and Computational Biology, Dana-Farber Cancer Institute, Harvard Medical School, Boston, \\ Massachusetts, USA. ${ }^{5}$ Clinical Research Development and Phase I Unit, CREA Laboratory, ASST Spedali Civili di Brescia, Brescia, BS, Italy. ${ }^{6}$ Comprehensive Cancer Center, Mayo Clinic, Scottsdale, Arizona, USA.
}

\begin{abstract}
Despite significant advances in the treatment of multiple myeloma (MM), most patients succumb to disease progression. One of the major immunosuppressive mechanisms that is believed to play a role in myeloma progression is the expansion of regulatory T cells (Tregs). In this study, we demonstrate that myeloma cells drive Treg expansion and activation by secreting type 1 interferon (IFN). Blocking IFN $\alpha$ and $\beta$ receptor 1 (IFNAR1) on Tregs significantly decreases both myeloma-associated Treg immunosuppressive function and myeloma progression. Using syngeneic transplantable murine myeloma models and bone marrow (BM) aspirates of MM patients, we found that Tregs were expanded and activated in the BM microenvironment at early stages of myeloma development. Selective depletion of Tregs led to a complete remission and prolonged survival in mice injected with myeloma cells. Further analysis of the interaction between myeloma cells and Tregs using gene sequencing and enrichment analysis uncovered a feedback loop, wherein myeloma-cell-secreted type 1 IFN induced proliferation and expansion of Tregs. By using IFNAR1-blocking antibody treatment and IFNAR1-knockout Tregs, we demonstrated a significant decrease in myeloma-associated Treg proliferation, which was associated with longer survival of myeloma-injected mice. Our results thus suggest that blocking type 1 IFN signaling represents a potential strategy to target immunosuppressive Treg function in MM.
\end{abstract}

\section{Introduction}

Immunotherapy has shown promising results in clinical trials against both solid (1) and hematological malignancies (2), such as Hodgkin lymphoma (3). Even though targeting the immune microenvironment can lead to significant improvements in response and patient survival, our understanding of its regulatory mechanisms is incomplete. The role of immunosurveillance in controlling cancer growth thus remains under active investigation in many cancer types, including multiple myeloma (MM).

$\mathrm{MM}$ is characterized by clonal expansion of malignant plasma cells in the bone marrow (BM). It is preceded by 2 precursor states, monoclonal gammopathy of undetermined significance (MGUS) and smoldering MM (SMM) (4), which represent stages of tumor progression with no evidence of end-organ damage. Unfortunately, MM remains an incurable disease despite significant advances in treatment. Therefore, immunotherapies may offer a promising strategy that could lead to significant responses, especially in patients with poor-risk cytogenetics who do not benefit from standard-ofcare options or in patients at early stages of disease, like SMM. On the other hand, MM might not be as immunogenic as other cancers,

Authorship note: YK and OZ contributed equally to this work. Conflict of interest: The authors have declared that no conflict of interest exists Submitted: April 21, 2016; Accepted: March 13, 2018.

Reference information: J Clin Invest. 2018;128(6):2487-2499.

https://doi.org/10.1172/JCl88169. such as melanoma or lung cancer $(5,6)$. Therefore, a better understanding of the immune microenvironment within the BM niche is critical for the development of novel immune therapies for not just $\mathrm{MM}$, but other cancers that infiltrate the BM.

Recent success of immune checkpoint inhibitors indicates that antitumor $\mathrm{T}$ cell responses are functionally inhibited by poorly understood immunosuppressive mechanisms (7). One of the major immunosuppressive mechanisms in tumor progression is the expansion of regulatory immune cells, particularly regulatory T cells (Tregs). Tregs are a subset of $\mathrm{CD}^{+} \mathrm{T}$ lymphocytes characterized by the expression of transcription factor forkhead box P3 (FOXP3) (8). They play an important role in the maintenance of self-tolerance and the control of immune homeostasis; however, they can be coopted by cancer cells to aid in immune suppression and evasion. Tregs can inhibit tumorspecific $\mathrm{CD}^{+}$and $\mathrm{CD}^{+} \mathrm{T}$ cell effector functions through cell-cell contact and/or the production of antiinflammatory cytokines. Additionally, they can induce effector T cell exhaustion, which is frequently seen in the tumor microenvironment (9).

In $\mathrm{MM}$, the role of Tregs in regulating tumor progression remains under active investigation $(10,11)$. Despite controversy in previous reports regarding the number and function of Tregs in the peripheral blood of MM patients (10-13), there is growing evidence supporting a role for Tregs in MM progression $(13,14)$. Recent data have shown a link between Tregs and sustenance of BM plasma cells, indicating that Treg activity directly affects homeostasis of the plasma cell population (15), while in vitro experiments have 
A
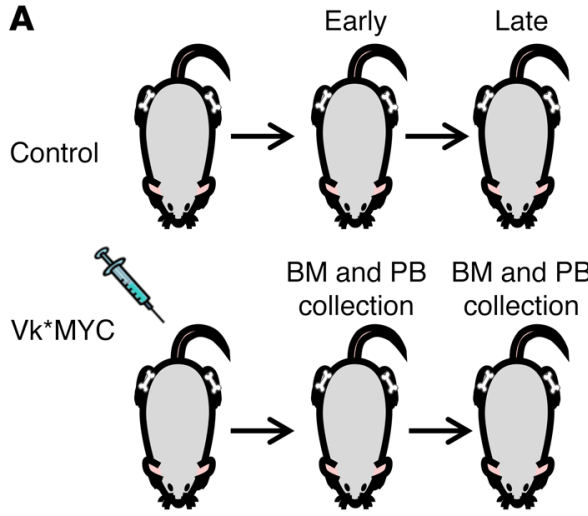

C
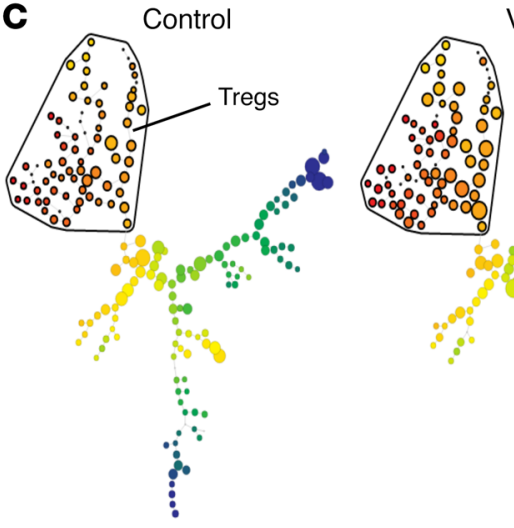

Vk*MYC

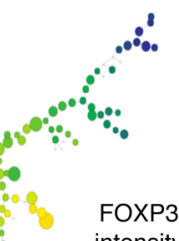

FOXP3
intensity

$\begin{array}{lll}0 & 0 \\ 0 & 0 & \end{array}$
B

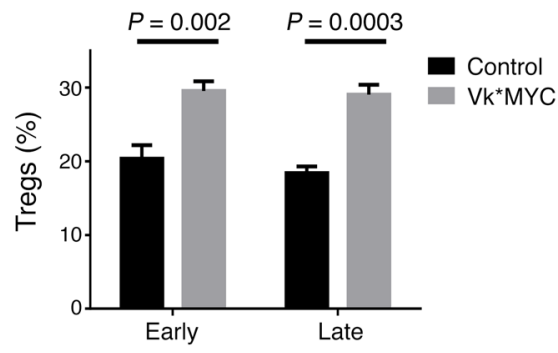

Figure 1. Tregs are increased in the BM of Vk*MYC transplantable mouse model.

(A) BM and PB were harvested from control $(n=3)$ and $\mathrm{Vk}^{*}$ MYC-injected mice $(n=$ 3) at early (day 14) and late (day 28) time points for CyTOF analysis. (B) Significant increase of Treg frequency within CD4 ${ }^{+}$ T-cells in the BM of Vk*MYC-injected mice compared with control mice from the early time point. Data validated by FACS $(n=5)$. (C) Spanning-tree progression analysis of density-normalized events (SPADE) was conducted on late BM CD4 ${ }^{+} T$ cells of control and $V k^{*} M Y C-i n j e c t e d$ mice. The size of the nodes indicates the frequency of each population, while the color indicates the expression of FOXP3. Increase of FOXP3 cells within $\mathrm{CD}^{+} \mathrm{T}$ cells was observed in $\mathrm{V}^{*}$ MYC-injected BM. (D) Significant increase in Treg frequency within CD4 ${ }^{+} \mathrm{T}$ cells in the PB of $\mathrm{Vk}^{*} \mathrm{MYC}$-injected mice compared with control mice at the late time point. (E) Significant decrease in the ratio of Teffs (CD4 ${ }^{+} \mathrm{CD} 44^{++} \mathrm{CD}_{2} \mathrm{~L}^{10}$ and $\left.\mathrm{CD} 8{ }^{+} \mathrm{CD} 44^{++}{ }^{+} \mathrm{CD} 62 \mathrm{~L}^{10}\right)$ to Tregs was observed in BM of $\mathrm{Vk}^{*} \mathrm{MYC}$ injected mice at the late time point as compared with $\mathrm{BM}$ of control mice. (F) Significant increase of $\mathrm{CD} 4^{+} \mathrm{CD} 25^{+} \mathrm{FOXP3}^{+}$cells and $\mathrm{CD}^{+} \mathrm{CD}^{2} 5^{+} \mathrm{FOXP3}^{+} \mathrm{CD} 127^{-10}$ Tregs in BM aspirates of SMM patients $(n=17)$ compared with healthy donors $(n=11)$. Data combined from 3 independent experiments. Cell numbers were normalized to the cell proportion in healthy BM (norma BM, NBM). $P$ values determined by 2-tailed $t$ test. Error bars indicate SD.
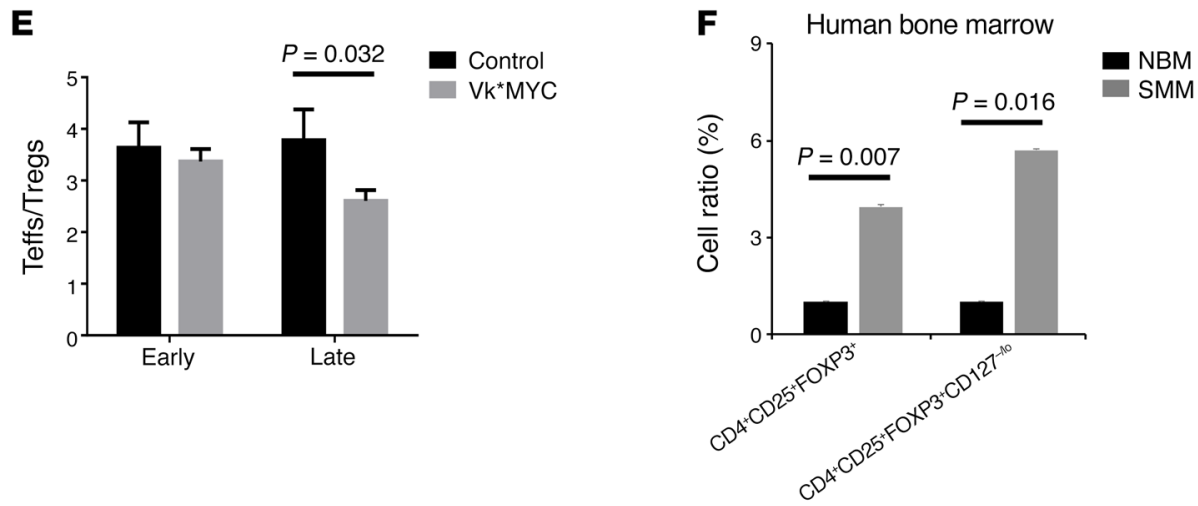

demonstrated that myeloma cells are capable of inducing Treg activation, predominantly in a contact-dependent manner $(16,17)$. Moreover, CD38-expressing Tregs have been suggested as suppressive modulators of antitumor immune responses in MM, while CD38 targeting has been shown to inhibit immunosuppression in ex vivo cocultures (17). In this context, understanding Treg homeostasis, and specifically how to overcome their suppressive function, is critical in developing effective immune therapies for myeloma and other hematological malignancies infiltrating the BM.

In the present study, we demonstrate that myeloma cells are responsible for Treg expansion and activation via secretion of type 1 interferon (IFN). Using the $\mathrm{Vk}^{*} \mathrm{MYC}$ transplantable mouse model, we show that Tregs are critical for MM disease progression and that depletion of Tregs leads to a complete remission. To further validate our findings, we show that blocking IFN $\alpha$ and $\beta$ receptor 1 (IFNAR1) signaling significantly decreases myeloma-associated Treg proliferation and activation, which is associated with prolonged survival of myeloma-injected mice. Our results thus suggest that blocking type 1 IFN signaling represents a potential immune-therapeutic strategy in MM.

\section{Results}

Increased number of Tregs at early stages of disease progression in the $B M$ of a transgenic MM transplantable mouse model and BM aspirates of MM patients. To examine the $\mathrm{T}$ cell immune microenvironment in MM during different stages of disease progression, we injected C57BL/6 mice with transplantable $\mathrm{Vk}^{*}$ MYC cells (Vk12598 cells) (18). The $\mathrm{Vk}^{*} \mathrm{MYC}$ transgenic mouse uniquely models the natural 
A

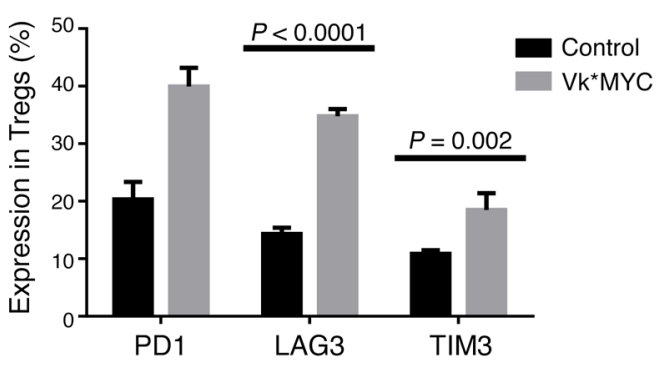

C

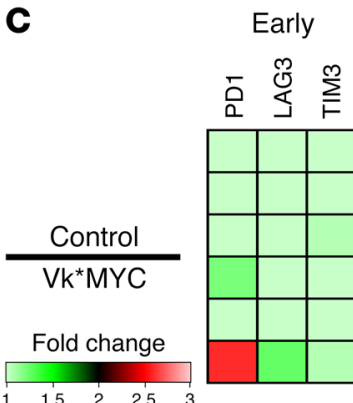

E

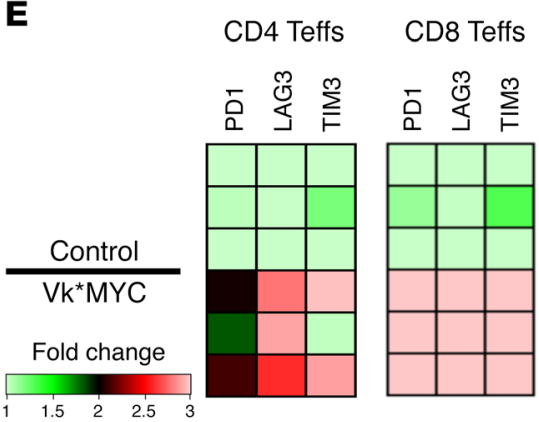

G

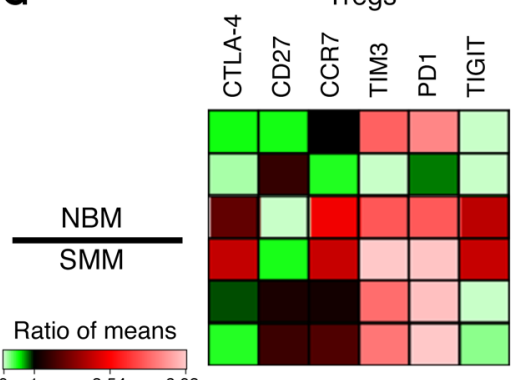

B Control

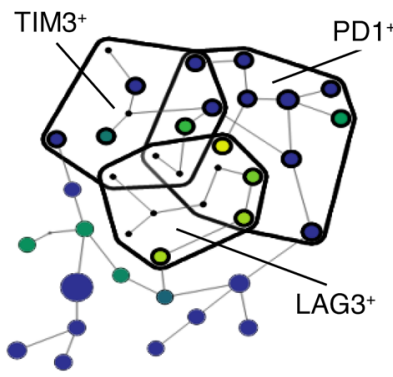

$\mathrm{Vk}^{*} \mathrm{MYC}$

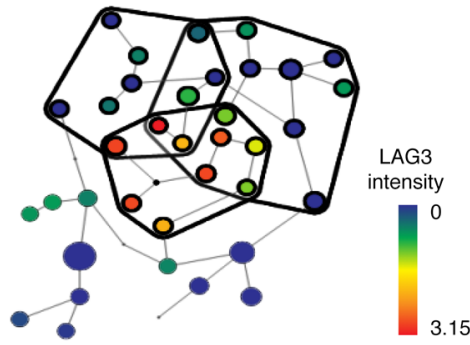

D

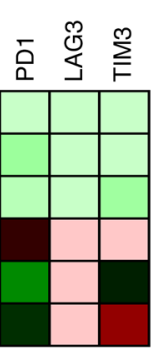

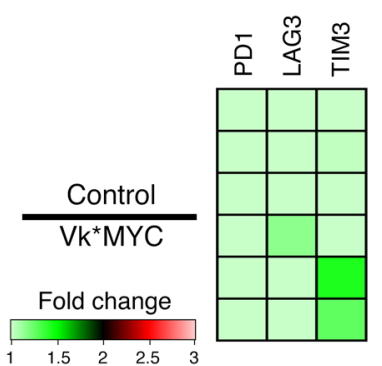

Late

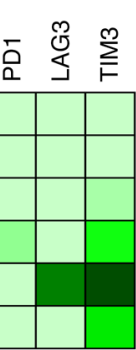

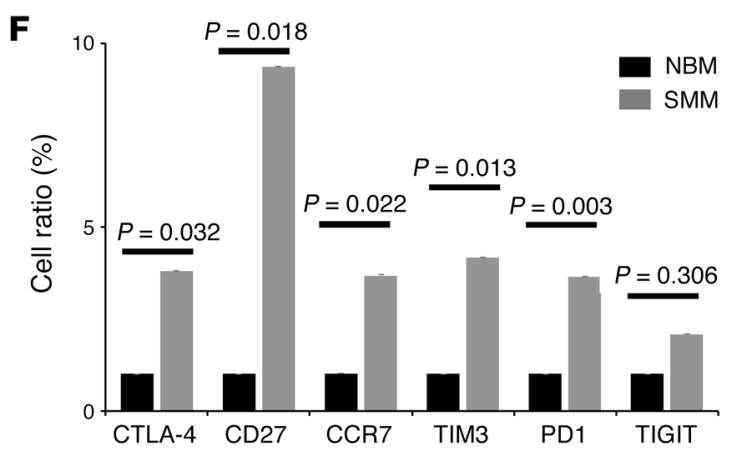

H
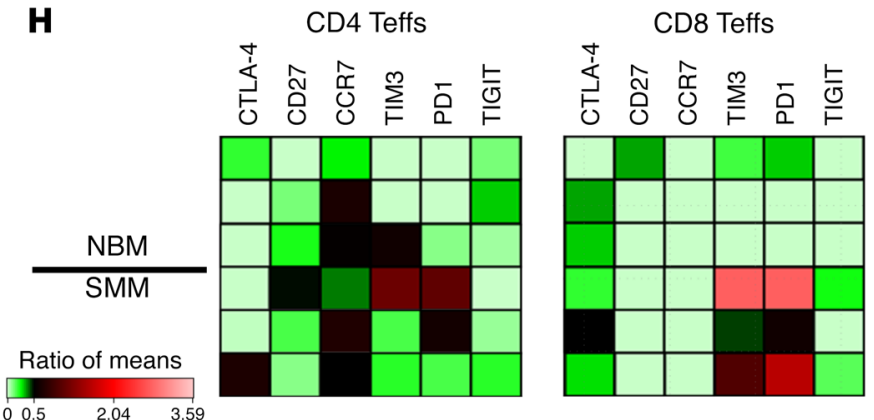

Figure 2. Upregulation of immune checkpoint molecules in Tregs in the BM of Vk*MYC-injected mice and SMM patients. (A) BM Tregs of Vk*MYC-injected mice have significantly higher frequency of immune checkpoint receptor-expressing (PD1, LAC3, and TIM3) Tregs compared with control BM Tregs at the late time point ( $n=3$ per group, validated by FACS with $n=5$ per group). (B) SPADE was conducted on BM Tregs of control and Vk*MYC-injected mice at the late time point. The nodes of the tree represent clusters of cells that are similar in marker expression. The color indicates the expression of LAG3. Increase of LAC3 ${ }^{+}$cells within Tregs was observed in Vk* MYC-injected BM. Heatmaps of immune checkpoint receptor (PD1, LAC3, and TIM3) expression of BM (C) and PB (D) Tregs at early and late time points. The color indicates the mean expression fold change of each molecule. BM Tregs of Vk*MYC-injected mice had higher expression of immune checkpoint receptors compared with control BM Tregs, while PB Tregs of Vk*MYC-injected mice did not show increased expression compared with control PB Tregs throughout disease progression. (F) Increased number of Tregs expressing immune checkpoint receptors in BM of SMM patients $(n=17)$ as compared with healthy donors $(n=11)$. Data combined from 3 independent experiments. NBM, normal bone marrow. (E) Heatmap of PD1, LAC3, and Tim3 expression on BM CD4 ${ }^{+}$and $C D 8^{+}$T effector cells (Teffs, $C D 44^{++} \mathrm{CD} 2 \mathrm{~L}^{10}$ ) at late time points. The color indicates the mean expression fold change of each molecule. BM Teffs of $\mathrm{Vk}^{*} \mathrm{MYC}$-injected mice had higher expression of immune checkpoint receptors compared with control BM Teffs. (G) Heatmap of immune checkpoint molecules and CCR7-chemokine receptor expression of Tregs in BM of MM patients compared with healthy donors. The color indicates the ratio of mean expression of each molecule. Tregs of MM patients show elevated expression levels of immune checkpoint molecules. (H) $\mathrm{CD}^{+}$and $\mathrm{CD8}{ }^{+} \mathrm{T}$ effector cells in BM of MM patients have higher expression of immune checkpoint molecules compared with healthy donors. The color indicates the ratio of mean expression of each molecule. $P$ values determined by 2-tailed $t$ test. Error bars indicate SD. 
history of human MM, with progression of disease from early premalignant stages to overt MM (19). To specifically investigate the changes that occur over time in the immune microenvironment within the $\mathrm{BM}$, where the tumor actually grows, versus peripheral blood (PB), we used mass cytometry (cytometry by time of flight [CyTOF]) (20) to analyze the immune cell profile of $\mathrm{Vk}^{*} \mathrm{MYC}$ injected mice, both in the $\mathrm{BM}$ and the $\mathrm{PB}$, at 2 time points during disease progression (days 14 and 28 after $\mathrm{Vk}^{*} \mathrm{MYC}$ injection) compared to control mice ( $n=3$ per group, per time point, Figure $1 \mathrm{~A})$. The gating strategy for Tregs is described in Supplemental Figure 1, A and B (supplemental material available online with this article; https://doi.org/10.1172/JCI88169DS1). CyTOF data were validated by flow cytometric analyses in independent experimental settings ( $n=5$ per group).

We observed a significant increase in the proportion of Tregs $\left(\mathrm{CD}^{+} \mathrm{FOXP3}^{+}\right)$in the $\mathrm{PB}$ and $\mathrm{BM}$ of $\mathrm{Vk}^{*} \mathrm{MYC}$-injected mice, compared with control mice. Interestingly, the differences were observed earlier in the BM starting from the early time point (Figure 1, B and C and Supplemental Figure 1, B-F), but only became detectable in the $\mathrm{PB}$ at the late time point (Figure 1D), indicating that Treg regulation occurs early within the tumor microenvironment, before these changes are reflected in the PB.

We then analyzed the ratio of effector $\mathrm{T}$ cells (Teffs: $\mathrm{CD} 4{ }^{+} \mathrm{CD} 44^{++} \mathrm{CD} 62 \mathrm{~L}^{\text {lo }}$ and $\left.\mathrm{CD} 8{ }^{+} \mathrm{CD} 44^{++} \mathrm{CD} 62 \mathrm{~L}^{\text {lo }}\right)(21,22)$ to Tregs to assess the suppression of $\mathrm{T}$ cell immunity in the BM microenvironment. We observed a decrease in the Teff/Treg ratio in the $\mathrm{BM}$ microenvironment and $\mathrm{PB}$ at the late time point (Figure 1E and Supplemental Figure 2A), suggesting the suppression of $\mathrm{T}$ cell immunity at a more advanced stage of disease.

Previous reports have shown an increased frequency of functional FOXP3-expressing $\mathrm{T}$ cells in the $\mathrm{PB}$ and $\mathrm{BM}$ of myeloma patients $(10,23)$. Here, we sought to define whether the increase in Tregs occurs in the BM already at the precursor stage of MM, i.e., smoldering multiple myeloma (SMM). For this, we analyzed the distribution of Tregs in BM aspirates of SMM patients $(n=$ $17)$, and compared it to that of healthy BM donors $(n=11)$. CyTOF analyses of the CD138-negative BM fractions demonstrated a significant increase of $\mathrm{CD}^{+} \mathrm{CD} 4^{+} \mathrm{T}$ cells (data not shown), activated $\mathrm{CD}^{+}{ }^{+} \mathrm{CD} 25^{+} \mathrm{FOXP}^{+}{ }^{+}$ceells, aswellas CD $4^{+} \mathrm{CD} 25^{+} \mathrm{FOXP}^{+} \mathrm{CD} 127^{-/ 10}$ Tregs within the $\mathrm{CD}_{4} 5^{+} \mathrm{CD}^{+}$compartment in SMM BM compared with healthy controls (Figure $1 \mathrm{~F}$ and Supplemental Figure 2B). These data from the human samples is in agreement with the murine transplantation model.

Immune checkpoint receptors are upregulated in Tregs present within the BM microenvironment of myeloma-injected mice. Immune checkpoint receptors, such as programmed cell death 1 (PD1), lymphocyte activation gene 3 (LAG3), and T cell immunoglobulin mucin 3 (TIM3), inhibit Teff function in the presence of cognate ligands (24). Conversely, when these receptors are expressed on Tregs, the function and/or proliferation of Tregs are enhanced (7). We measured the number of positive cells, as well as the mean expression of immune checkpoint receptors (PD1, LAG3, and TIM3) on Tregs in the BM and PB of $\mathrm{Vk}^{*} \mathrm{MYC}$-injected and control mice by CyTOF. BM Tregs of myeloma-bearing mice showed significantly higher expression of immune checkpoint receptors, compared with control BM Tregs (Figure $2, \mathrm{~A}-\mathrm{C}$ ). In contrast, $\mathrm{PB}$ Tregs of $\mathrm{Vk}^{*} \mathrm{MYC}$-injected mice did not show increased expression of these receptors, compared with control PB Tregs (Figure 2D and Supplemental Figure 3, A-C), suggesting that tumor site affects Treg phenotype and enhances its suppressive function. Spanningtree progression analysis of density-normalized events (SPADE), as depicted in Figure 2B, revealed a significant increase in LAG3 expression on BM Tregs expressing PD1 and/or TIM3; LAG3 has been shown to be a marker of Tregs expanding at the tumor site (25). Along with the increase in proportion of Tregs expressing immune checkpoint receptors in BM of myeloma-injected mice, there is an elevated number of Tregs coexpressing PD1, LAG3, and TIM3 receptors as compared with healthy mice (Supplemental Figure 3D). Interestingly, we also observed significantly higher levels of expression of PD1, LAG3, and TIM3 on Vk $\mathrm{Vk}^{*} \mathrm{MC}$-injected BM $\mathrm{CD}^{+}$and $\mathrm{CD}{ }^{+}$Teffs $\left(\mathrm{CD} 44^{++} \mathrm{CD} 62 \mathrm{~L}^{\mathrm{lo}}\right)$, which represent markers of $\mathrm{T}$ cell exhaustion and suppressed effector functions (Figure $2 \mathrm{E}$ and Supplemental Figure 3, E-G). These results were further validated in a second mouse model of MM, C57BL/KaLwRij, that was injected with 5TGM1 murine myeloma cells. Similar to the results of the $\mathrm{Vk}^{*} \mathrm{MYC}$-injected mice, we observed a significant increase in the frequency and activation of Tregs in the BM microenvironment of 5TGM1-injected C57BL/KaLwRij mice, compared with the control group (Supplemental Figure 4, A-D).

In line with our murine data, we observed a significantly higher proportion of $\mathrm{CD}^{+} \mathrm{CD} 25^{+} \mathrm{FOXP}^{+}$Tregs expressing immune checkpoint molecules in BM aspirates of SMM patients $(n=17)$ compared with those in healthy BM $(n=11)$ (Figure $2 \mathrm{~F})$. Interestingly, SMM patients have significantly higher numbers of C-C chemokine receptor type 7-expressing (CCR7-expressing) cells in their $\mathrm{CD}^{+}{ }^{+} \mathrm{CD} 25^{+} \mathrm{FOXP}^{+}$Treg compartment, which indicates an upregulation of the migratory phenotype of FOXP3-expressing cells (Figure 2F). Examination of immune molecules' coexpression on Tregs revealed that mainly the proportion of cells expressing PD1, TIM3, and CTLA4 was significantly increased in the SMM group (Supplemental Figure 4E). In addition, analysis of the mean expression fold change of each immune checkpoint molecule indicates elevated levels of immune checkpoint receptors on Tregs in individual SMM patients as compared with healthy donors (Figure 2G). Further analyses of the CyTOF profiles of immune checkpoint molecules present on $\mathrm{CD} 4^{+} \mathrm{Teffs}$ and $\mathrm{CD}^{+}$Teffs in the BM of SMM patients, compared with healthy $\mathrm{BM}$, revealed individual patients with elevated expression of TIM3, PD1, CTLA-4, and CCR7, as depicted in the heatmap data in Figure $2 \mathrm{H}$. In $35.3 \%$ of the SMM patients, we observed at least a 2-fold increase in the levels of CD27 and CCR7 on CD $4{ }^{+}$Teffs and TIGIT on CD8 ${ }^{+}$Teffs as compared with the expression of these molecules in the BM of healthy donors. Together, these results indicate an upregulated expression of immune checkpoint inhibitors on Tregs within the myeloma BM microenvironment as early as the SMM stage.

Tregs contribute to $V k^{*} M Y C$ progression. A CD38-expressing subset of Tregs from MM patients has been shown to exhibit immunosuppressive activity in ex vivo cocultures (17). In order to determine the significance of Tregs in myeloma progression in vivo, we used a transgenic inducible knockout (KO) animal model, depletion of regulatory T cell (DEREG) mice, expressing a diphtheria toxin (DT) receptor-enhanced green fluorescent protein (EGFP) fusion protein under the control of the FOXP3 gene 
A
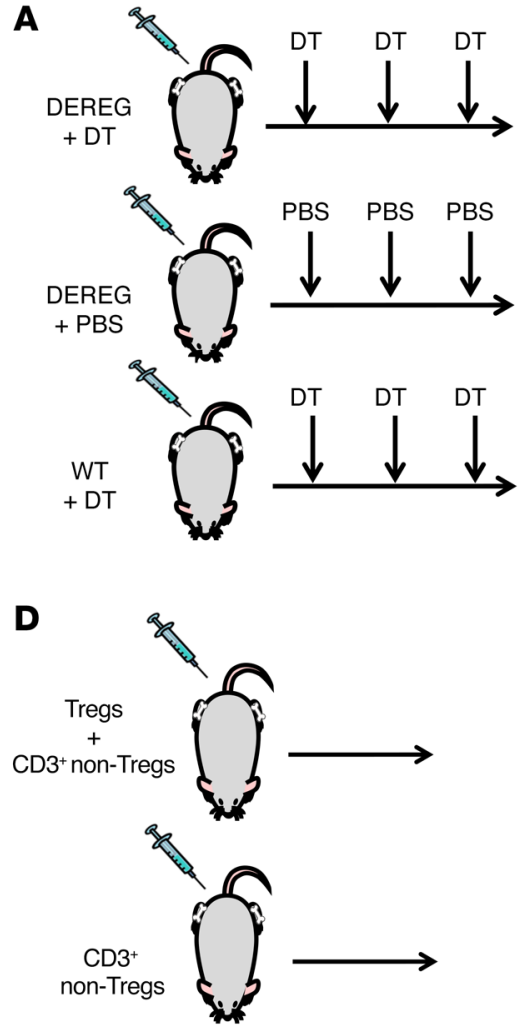

B

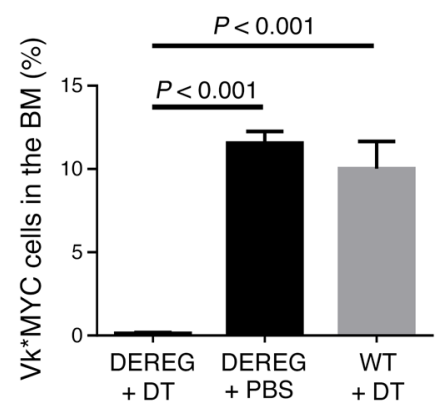

E - Tregs $+\mathrm{CD}^{+}$non-Tregs

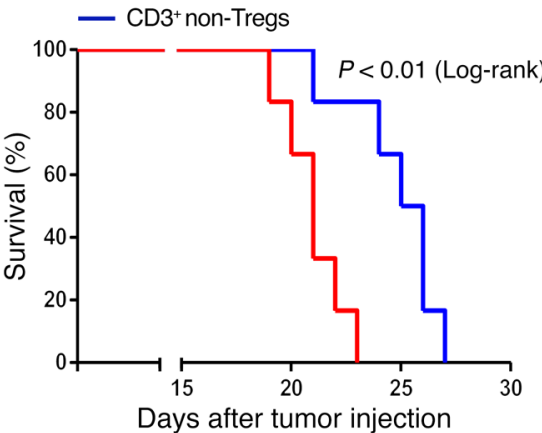

C

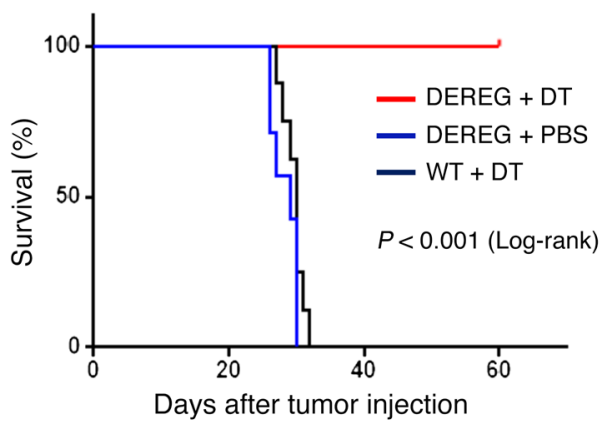

$\mathbf{F}$

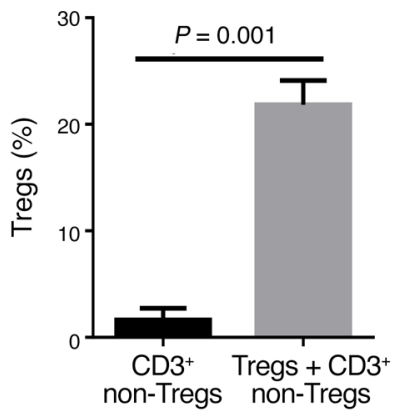

Figure 3. Treg depletion in DEREG mice leads to extended survival of Vk*MYC-injected mice, while adoptive transfer of Tregs promotes tumor progression in vivo. (A) Diphtheria toxin (DT) or PBS was injected 3 times (day 5, day 12, and day 19) after Vk*MYC injection into DEREC mice or wild-type mice ( $n=7 /$ group). (B) Proportion of Vk* MYC cells (CD138+B220 cells) in the BM of each group at day 20 after $\mathrm{Vk}^{*}$ MYC injection. Three mice from each group were sacrificed at day 20 for analysis of BM Vk*MYC frequency. Significant tumor reduction occurred in the DEREG mice treated with DT compared with control mice. $P$ values determined by 2-tailed $t$ test with Bonferroni's correction. (C) Kaplan-Meier curve showing significant increase in survival of DEREC mice treated with DT (DEREG + DT) compared with control groups (DEREG + PBS, wild type + DT) ( $n=7 /$ group). $P<0.0001$ by log-rank test. (D) For Treg transfer experiments, $3 \times 10^{6}$ non-Tregs were injected with or without $1 \times 10^{6}$ Tregs i.v. into Rag2-KO mice followed by Vk* MYC cell injection 5 days afterwards. (E) Kaplan-Meier curve showing mice injected with Tregs and CD3+ non-Tregs (Tregs + $C D 3^{+}$non-Tregs: median survival 21 days) had significantly shorter survival compared with mice injected only with $\mathrm{CD}^{+}$non-Tregs ( $\mathrm{CD3}^{+}$non-Tregs: median survival 25.5 days) $(n=6 /$ group). $P<0.01$ by log-rank test. (F) FACS analysis of mice BM showing significant increase of BM Tregs in the Tregs $+C D 3^{+}$non-Tregs group compared with $C D 3^{+}$non-Tregs group $(n=3 /$ group). $P$ value determined by 2 -tailed $t$ test. Error bars indicate SD.

locus, that allows the selective depletion of FOXP3 ${ }^{+}$Tregs by DT injection (26). Survival of DEREG mice injected with $\mathrm{Vk}^{*} \mathrm{MYC}$ cells, followed by 3 injections of DT, was compared to that of $\mathrm{Vk}^{*} \mathrm{MYC}$-injected DEREG mice injected with PBS and that of $\mathrm{Vk}^{*} \mathrm{MYC}$-injected wild-type littermates injected with DT (Figure 3A). Effective depletion of BM Tregs in $\mathrm{Vk}^{*} \mathrm{MYC}$-injected mice was observed upon DT injection into DEREG mice (Figure 3B). A significant tumor reduction was observed in the DT-treated DEREG mice, compared with control groups, as shown by the number of $\mathrm{CD} 138^{+} \mathrm{B} 220^{-} \mathrm{Vk}^{*} \mathrm{MYC}$ cells obtained from their BMs (Supplemental Figure 5A). More importantly, there was a significant difference in survival, with the control group surviving for a median of 30 days, while the DT-treated DEREG mice showed no evidence of myeloma progression for up to 60 days of experimental setup $(P<0.0001)$ (Figure $3 C)$. Flow cytometric analysis of $\mathrm{BM}$ cells on day 3 after the last DT treatment in $\mathrm{Vk}^{*} \mathrm{MYC}$-injected mice demonstrates a significant decrease in CD138-expressing plasma cells and an increased Teff/Treg ratio compared with the control group (Supplemental Figure 5B). Interestingly, CyTOF analysis of the cellular content of BM cells showed a significantly increased proportion of CD19-expressing B cells and F4/80+ macrophages in mice with Treg depletion compared with control mice (Supplemental Figure 5C).

To further confirm the critical importance of Tregs in regulating tumor progression in MM, we performed a Treg transfer experiment, in an effort to demonstrate that the adoptive transfer of Tregs will lead to rapid tumor progression in vivo. We injected sorted $\mathrm{C} 57 \mathrm{BL} / 6 \mathrm{CD}^{+} \mathrm{CD} 25^{-}$non-Tregs, with or without $\mathrm{CD} 4{ }^{+} \mathrm{CD} 25^{+}$Tregs, into RAG2-KO immune-deficient mice, which lack a mature $\mathrm{T}$ and $\mathrm{B}$ cell compartment, followed by $\mathrm{Vk}^{*} \mathrm{MYC}$ transplantation, and measured their survival (Figure 3D). In the short time period of approximately 30 days of survival, we did not observe weight loss or signs of colitis in these mice. We also did not observe diarrhea or GI symptoms in these mice. Mice injected with Tregs and $\mathrm{CD}^{+}$non-Tregs had significantly shorter survival compared with mice injected with $\mathrm{CD}^{+}$non-Tregs only (Figure $3 \mathrm{E}$ ), indicating that the Treg compartment suppresses antimyeloma $\mathrm{T}$ cell immunity. Analysis of the $\mathrm{BM}$ of moribund mice 


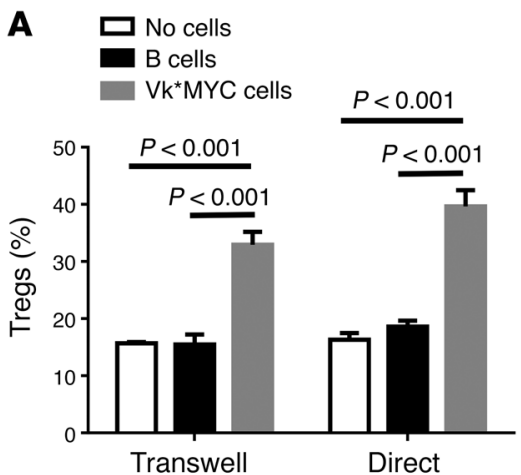

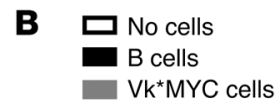

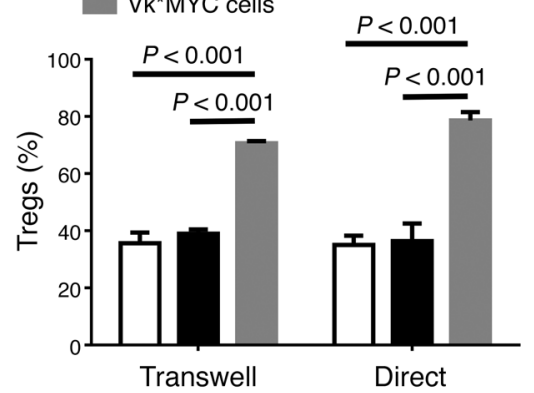

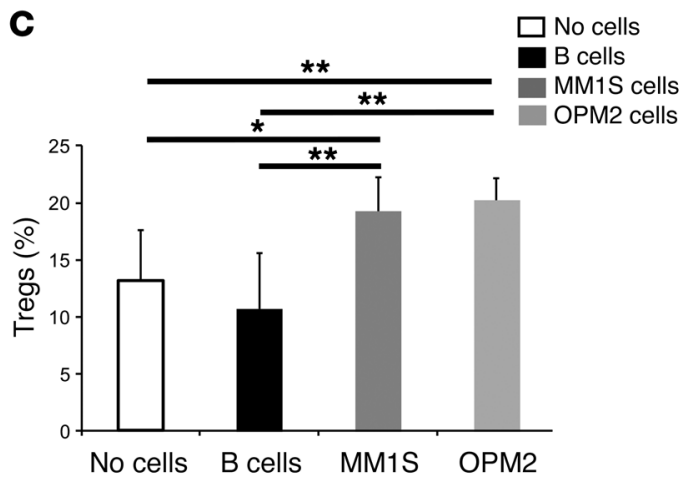

Figure 4. Myeloma cells induce and expand Tregs in vitro. (A) Treg induction assay. CD4+CD25- non-Tregs were cocultured with $\mathrm{C57BL} / 6 \mathrm{~B}$ cells or $\mathrm{Vk}{ }^{*} \mathrm{MYC}$ cells for 72 hours using transwell or direct coculture. Significant Treg induction was observed in the $\mathrm{Vk}^{*} \mathrm{MYC}$ coculture group either under transwell or direct coculture compared with the no-cells or B cells group. Average of experiments performed in triplicate is shown. The experiment was repeated 3 times. (B) Treg expansion assay. CD4+CD25+ Tregs were cocultured with C57BL/6 B cells or Vk* MYC cells for 72 hours using transwell or direct coculture. FOXP3 ${ }^{+}$cells within the CD4+ cells are shown. Significant Treg expansion was observed in the $\mathrm{Vk}^{*} \mathrm{MYC}$ coculture group either under transwell or direct coculture compared with the no-cells or B cells group. Average of experiments performed in triplicate is shown. The experiment was repeated 3 times. (C) Significant Treg induction was observed in coculture of MM1S and OPM2 myeloma cells with human PB cells. Human CD4+CD25- cells were cocultured with MM1S or OPM2 myeloma cells for 72 hours using transwell coculture. MM1S and OPM2 coculture groups were compared to the no-cells or CD19-expressing B cells group. Treg cells were assessed as $\mathrm{CD} 4{ }^{+} \mathrm{CD} 25^{+} \mathrm{FOXP3}^{+}$cells. Data from independent experiments performed in triplicate of 3 different $\mathrm{PB}$ donors is shown. ${ }^{*} P<0.05$; ${ }^{*} P$ $<0.01$ by 2 -tailed $t$ test with Bonferroni's correction. Error bars indicate SD.

showed significant increase of Tregs in the group injected with $\mathrm{CD} 4^{+} \mathrm{CD} 25^{+}$Tregs (Figure $3 \mathrm{~F}$ and Supplemental Figure 5D), confirming that transferred Tregs proliferated in the recipient's BM tumor microenvironment.

In parallel, to further validate our results, we specifically depleted Tregs in $\mathrm{Vk}^{*} \mathrm{MYC}$-injected mice using an anti-CD25 antibody ( $250 \mu \mathrm{g}$ of anti-murine CD $25 \mathrm{mAb}$ on day 4 before [day -4] and day 10 after $\mathrm{Vk}^{*} \mathrm{MYC}$ cell injection, $n=6$ ); isotype antibody treatment was used in the control group $(n=6)$, as described in previous studies (27). Compared with isotype-treated mice, the anti-CD25-treated group demonstrated significantly longer survival (Supplemental Figure 6, A and B), further confirming the critical role of Tregs in myeloma progression. Together, our data provide direct in vivo evidence of Tregs supporting MM disease progression.

Human and murine myeloma cells induce and expand Tregs in a contact-dependent and-independent manner. To elucidate mechanisms involved in Treg induction and expansion in MM, we performed Treg induction and expansion assays by coculturing $\mathrm{Vk}^{*} \mathrm{MYC}$ cells with $\mathrm{CD}^{+} \mathrm{CD} 25^{-}$non-Tregs or $\mathrm{CD} 4^{+} \mathrm{CD} 25^{+}$ Tregs, respectively, using direct coculture assays. Both assays showed significant increase in $\mathrm{CD} 4{ }^{+} \mathrm{CD} 25^{+} \mathrm{FOXP} 3^{+}$Tregs when cocultured with $\mathrm{Vk}^{*} \mathrm{MYC}$ cells, compared with B cells or no-cell controls (Figure 4, A and B). Similar results were obtained when a transwell coculture assay separating $\mathrm{Vk}^{*} \mathrm{MYC}$ cells from $\mathrm{T}$ cells was performed, indicating that it is a soluble factor produced from $\mathrm{Vk}^{\star} \mathrm{MYC}$ cells that leads to the induction and expansion of Tregs (Figure 4, A and B and Supplemental Figure 7A).

We further explored a contact-independent Treg induction mechanism in human samples. For this, we cocultured human $\mathrm{CD} 4{ }^{+} \mathrm{CD} 25^{-} \mathrm{PB}$ cells with MM1S and OPM2 myeloma cell lines without cell-cell interactions. Consistent with our murine data, we observed a significant induction of $\mathrm{CD}^{+} \mathrm{CD} 25^{+} \mathrm{FOXP}^{+}$cells by coculture of $\mathrm{CD} 4^{+} \mathrm{CD} 25^{-} \mathrm{PB}$ cells with myeloma cells, compared with CD19-expressing B cells or no-cell controls (Figure 4C).
Together, these data show that murine and human myeloma cells induce and promote expansion of Tregs in a cell-cell contactdependent and -independent manner, leading to the increased presence of Tregs in the tumor microenvironment.

Myeloma cells regulate Tregs in the BM microenvironment by secretion of type 1 IFNs. To further characterize mechanisms that lead to the activation of myeloma-associated Tregs, we performed RNA sequencing (RNA-seq) of flow-sorted BM EGFP-expressing Tregs from $\mathrm{Vk}^{*} \mathrm{MYC}$-injected DEREG mice and control DEREG mice. We observed a significant difference in mRNA expression between Tregs derived from the BM microenvironment of myeloma-injected mice and those derived from control mice, as depicted in the heatmap in Figure 5A. The top genes that were significantly up- or downregulated in Tregs from $\mathrm{Vk}^{\star} \mathrm{MYC}$-injected mice, compared with control, are shown in Supplemental Table 1. Consistent with our CyTOF data at the protein level, expression of checkpoint-related molecules, including PDCD1 (PD1), LAG3, and HAVCR2 (TIM3), was significantly upregulated in $\mathrm{Vk}^{*} \mathrm{MYC}$ associated Tregs, compared with control Tregs (Figure 5B). Additionally, other checkpoint-related molecules, such as TNFRSF4 (OX40) (28) and TNFRSF18 (GITR) (29), were also found to be highly expressed in $\mathrm{Vk}^{*} \mathrm{MYC}$-associated Tregs (Figure $5 \mathrm{~B}$ ).

Tregs use multiple effector molecules, such as IL-10, TGF- $\beta$, EBI3, FGL2, GZMB, and PRF1, to suppress the immune effector cells (30-32). In our study, Vk* MYC-associated Tregs showed a significantly higher expression of EBI3, FGL2, and GZMB (Figure $5 \mathrm{C}$ ), indicating that these molecules may play a role in the suppressive activity of myeloma-associated Tregs.

Chemokine receptors play a significant role in Treg migration and homing. We analyzed the expression of CXCR3, CCR6, and CCR7, which are all well-known mediators of Treg migration to inflammatory sites (33-37). CXCR3 was found to be significantly overexpressed in $\mathrm{Vk}^{*} \mathrm{MYC}$-associated Tregs, compared with control (Figure 5D), suggesting an important role for CXCR3 
A

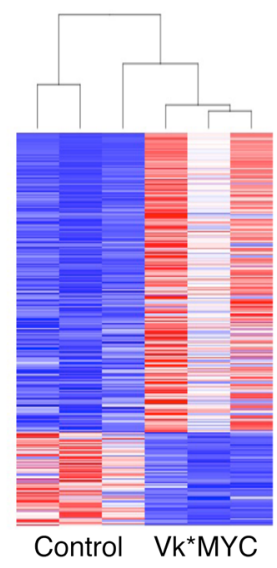

B

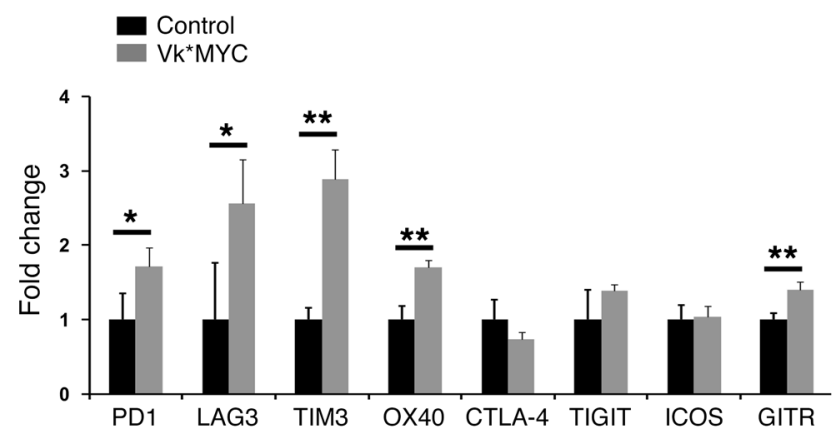

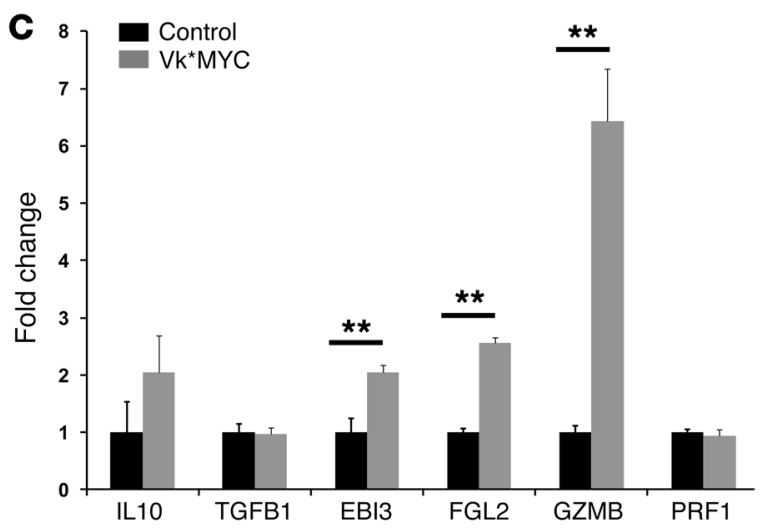

D Control

Vk*MYC

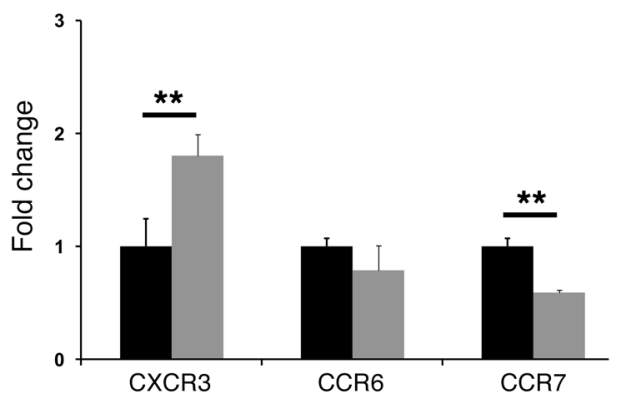

Figure 5. Differences in gene expression between myeloma-associated and control Tregs in the BM microenvironment. (A) mRNA expression profiling by RNA sequencing of total RNA isolated from control $(n=3)$ and Vk* MYC-injected $(n=3)$ BM Tregs. A heatmap was generated after supervised hierarchical cluster analysis. Differential mRNA expression is shown in red (upregulation) versus blue (downregulation) intensity (control versus Vk* MYC, 2-fold change, $P<0.05)$. (B) Differences in checkpoint-related gene expression levels of Tregs isolated from Vk* MYC-injected and control mice. A number of checkpointrelated molecules were significantly upregulated in Tregs from $\mathrm{Vk}^{*} \mathrm{MYC}$-injected mice compared with control Tregs. The gene expression fold change in Tregs from Vk*MYC-injected mice compared with those from control Tregs is shown. (C) Differences in Treg effector gene expression levels between V ${ }^{*} M Y C$ and control Tregs. The gene expression fold change of Tregs from $\mathrm{Kk}^{*} \mathrm{MYC}$-injected mice compared with control Tregs is shown. (D) Differences in chemokine receptor gene expression levels between Tregs from Vk ${ }^{*} M Y C$-injected mice and control Tregs. ${ }^{*} P \leq 0.05 ;{ }^{* *} P<0.01$ by 2 -tailed $t$ test. Error bars indicate SD.

in Treg migration to the myeloma microenvironment. Interestingly though, CCR7 levels were significantly downregulated in $\mathrm{Vk}^{*} \mathrm{MYC}$-associated Tregs, compared with control, indicating differences in the regulation of Treg migration in the tumor setting.

To determine pathways that regulate Treg activation and expansion in the MM tumor microenvironment, we performed gene set enrichment analysis (GSEA) for the genes that were found to be significantly upregulated in $\mathrm{Vk}^{*} \mathrm{MYC}$-associated Tregs, compared with control. Interestingly, GSEA showed enrichment of type $1 \mathrm{IFN}$-related genes in $\mathrm{Vk}^{*} \mathrm{MYC}$-associated Tregs (Figure 6A). According to the GSEA, several differentially regulated proteins contributed to the enrichment score for the IFN- $\alpha / \beta$ signaling pathway. These include the type $1 \mathrm{IFN}$-induced proteins playing a critical role in cellular innate antiviral responses, such as IFN-stimulated 15-kDa protein (ISG-15), Mx1, Mx2, IFIT1, IFIT3, OAS2, and OAS3. Additionally, key transcriptional regulators of type I IFN-dependent immune responses, such as IFN regulatory factors 7 and 9 (IRF7 and IRF9) contributed to the enrichment profile. Signal transducer and activator of transcription 1 (STAT1) and STAT2 were enriched in the gene list as well. These results indicate that the type 1 IFN pathway is activated in the BM Tregs of $\mathrm{Vk}^{*} \mathrm{MYC}$-injected mice and may constitute a novel pathway of Treg regulation in the MM immune microenvironment.

We then sought to examine whether myeloma cells secrete type 1 IFNs, potentially leading to Treg activation. We observed a significant increase of IFN- $\beta$ in the supernatants of $\mathrm{Vk}^{*} \mathrm{MYC}$ cells in culture, compared with the supernatant of control B cells obtained from C57BL/6 mice (Figure 6B), while IFN- $\alpha$ was below the detection level in both cell types (data not shown). Collectively, these results suggest that myeloma-cell-secreted type 1 INF could be a mediator of Treg activation in MM.

INFA1 expression is associated with poor outcome in MM patients. We then investigated whether CD138-expressing plasma cells from MM patients showed significant changes in pathways or the expression levels of genes related to type 1 IFN signaling. GSEA of 2 gene expression data sets generated from CD138-enriched plasma cells, one from 73 newly diagnosed MM patients and one from 147 cases of MGUS, SMM, MM, and relapsed myeloma (GSE6477), showed significant enrichment for type 1 IFN signaling compared with 15 healthy donors (Figure 6C). Notably, upreg- 
A

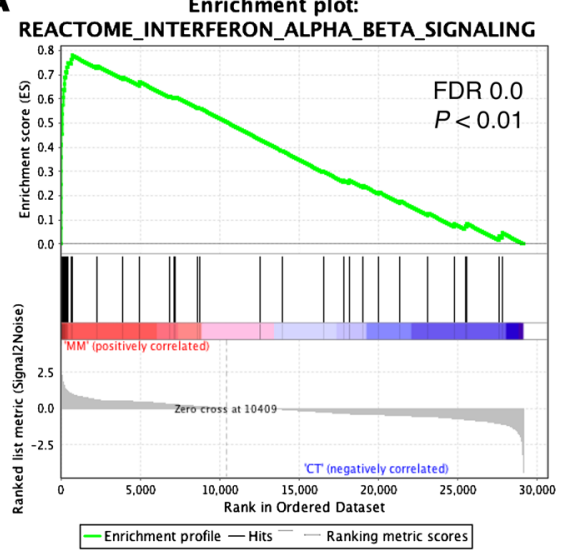

B

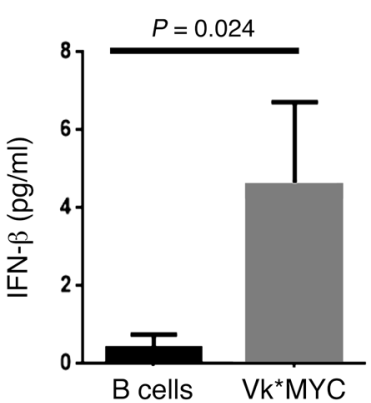

Enrichment plot: REACTOME INTERFERON_SIGNALING

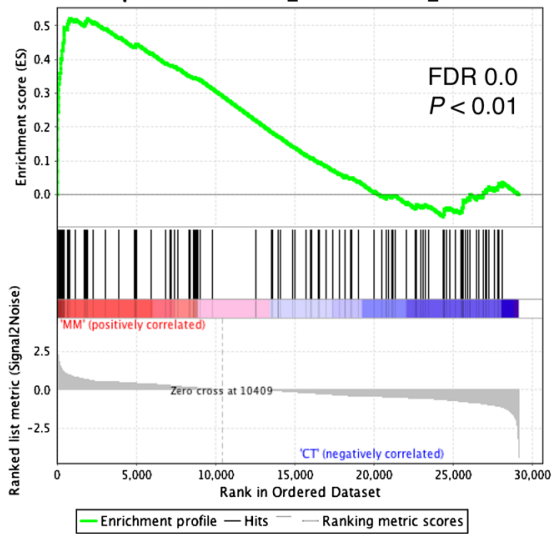

C

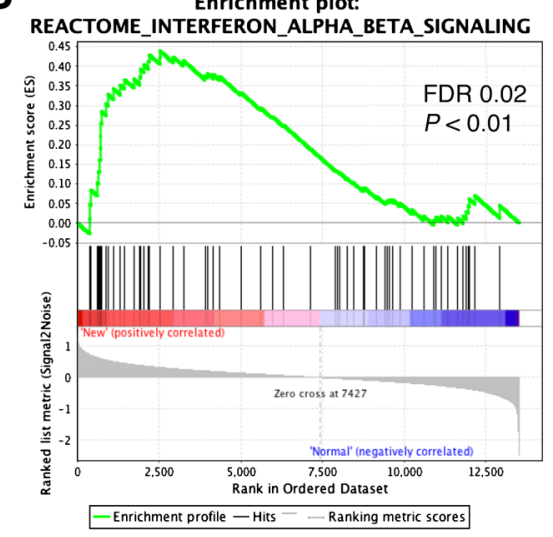

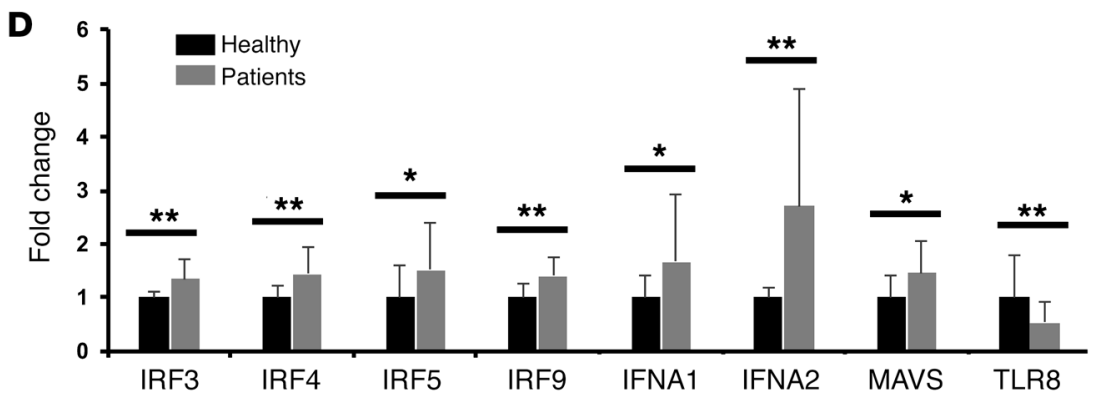

E

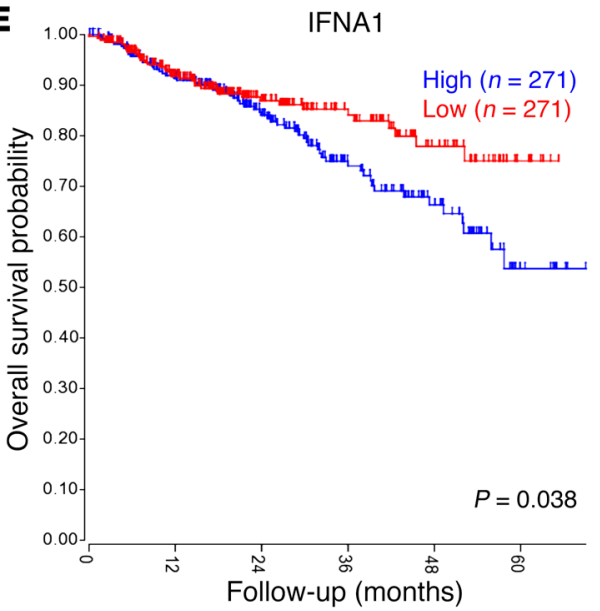

$\mathbf{F}$

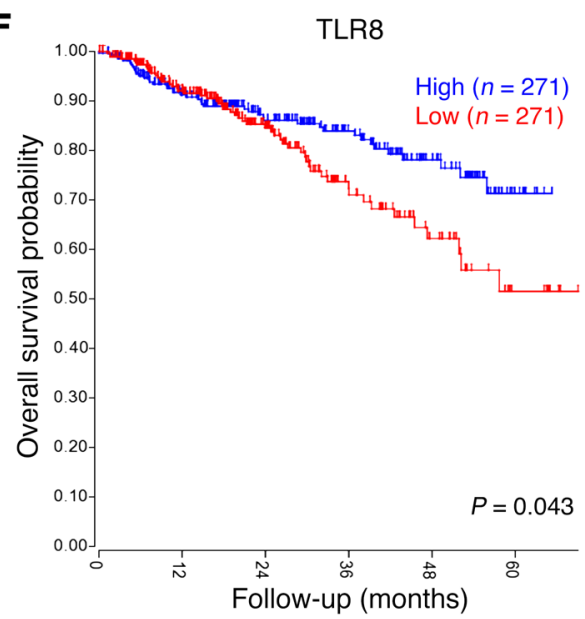

Figure 6. Type 1 IFN signaling associated with Treg activation and overall patient survival. (A) Vk* $M Y C$ BM Tregs show an enrichment for type 1 IFN-related mRNA signature compared with control BM Tregs, as presented by GSEA. The green curves show the enrichment score and reflect the degree to which each gene (black vertical lines) is represented at the top or bottom of the ranked gene list. The heatmap indicates the relative abundance (red to blue) of the genes specifically enriched in the $\mathrm{Vk}^{*} \mathrm{MYC}$ Tregs as compared with control Tregs. FDR and $P$ values and are shown for each gene set analyzed. (B) IFN- $\beta$ secretion from $V^{*} M Y C$ cells. IFN- $\beta$ secretion, detected by ELISA, in Vk* MYC cell culture supernatants was significantly higher compared with the supernatants of control B cells obtained from C57BL/6 mice. Average of experiments performed in triplicate is shown. The experiment was repeated 3 times. (C) Myeloma cells from MM patients show enrichment in type 1 IFN signaling pathway compared with cells from healthy donors. (D) Differential gene expression levels for genes involved in type 1 IFN production. (E) IFNA1 overexpression is associated with worse overall survival in MM patients. (F) Expression of TLR8 significantly correlates with worse outcome in MM patients. ${ }^{*} P<0.05$; ${ }^{*} P<0.01$ by 2 -tailed $t$ test. Error bars indicate SD. 
A

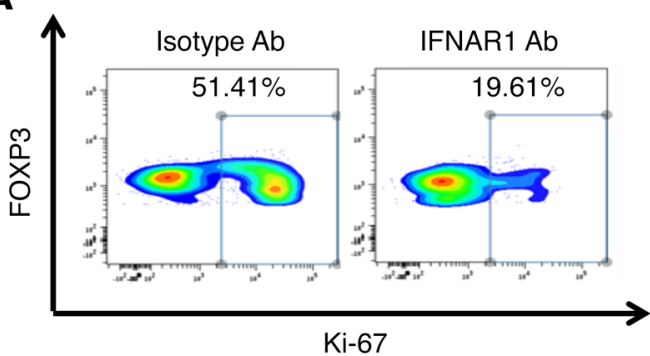

C

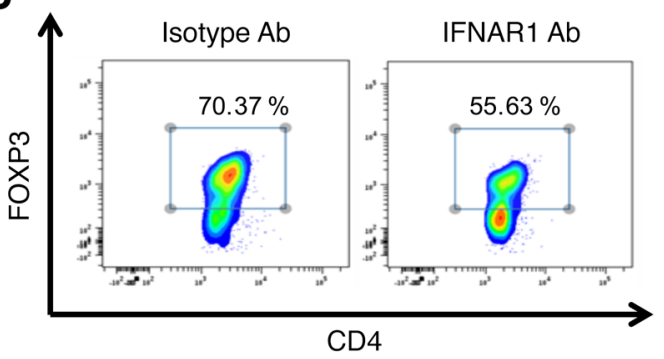

B

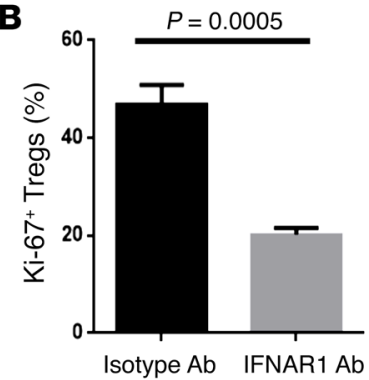

D

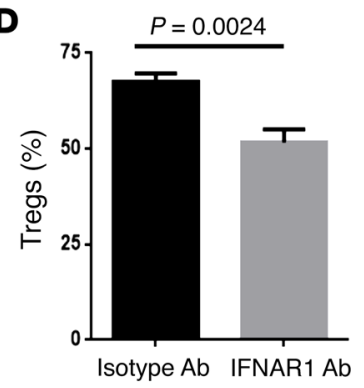

$\mathbf{E}$

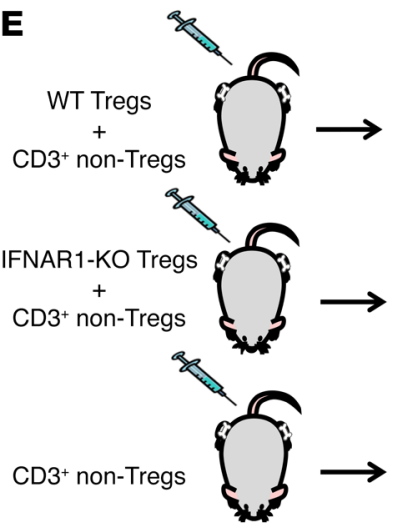

G

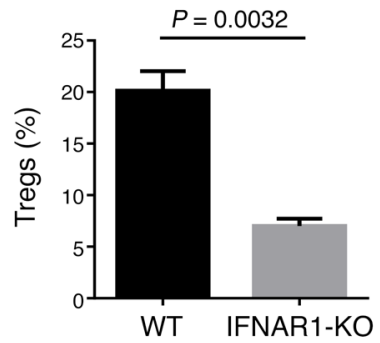

$\mathbf{F} \quad-W T$ Tregs $+C D 3^{+}$non-Tregs
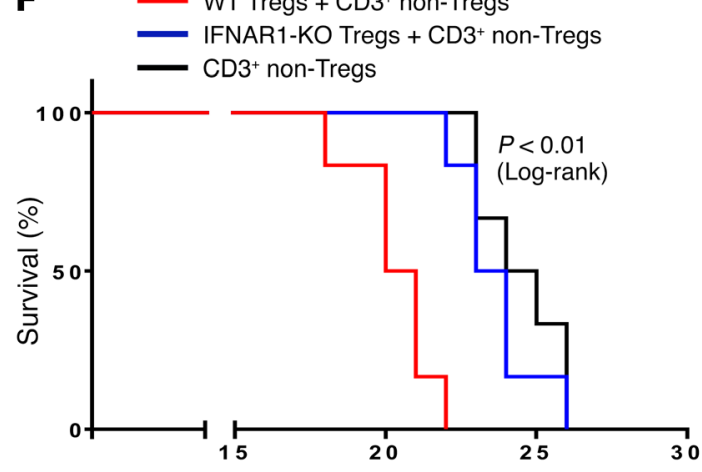

Days after tumor injection

H

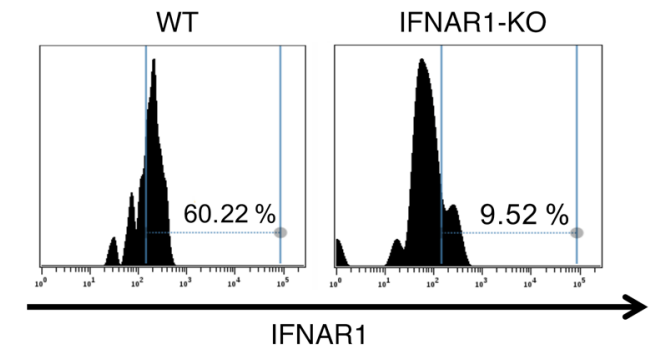

Figure 7. Blocking IFNAR1 suppresses Treg expansion and myeloma progression. (A) Representative FACS analysis of Ki-67 expression of Tregs expanded under coculture with Vk* MYC cells and IFNAR1 antibody (IFNAR1 Ab) or isotype control (isotype Ab). (B) Significant decrease of Ki-67 expression in Tregs cocultured with Vk* MYC and IFNAR1 Ab compared with those with isotype Ab. Average of experiments performed in triplicate is shown. The experiment was repeated 3 times. (C) Representative FACS analysis of Treg expansion assay with IFNAR1 Ab or isotype Ab coculture. Proportion of $\mathrm{FOXP3}^{+}$cells in the $\mathrm{CD} 4^{+}$cells is shown. (D) Significant decrease of Treg proliferation by cells cocultured with Vk* MYC and IFNAR1 Ab compared with isotype Ab. Average of experiments performed in triplicate is shown. The experiment was repeated 3 times. (E) Non-Tregs (3 $\left.\times 10^{6}\right)$ were injected with or without $1 \times 10^{6} \mathrm{C} 57 \mathrm{BL} / 6$ Tregs or IFNAR1-KO Tregs i.v. into Rag2-KO mice followed by Vk*MYC cell injection 5 days afterwards. (F) Kaplan-Meier curve showing survival of mice injected with C57BL/6 Tregs and CD3 ${ }^{+}$non-Tregs (WT Tregs + $\mathrm{CD3}^{+}$non-Tregs) compared with mice injected with IFNAR1-KO Tregs and CD3+ non-Tregs (IFNAR1-KO Tregs + $\mathrm{CD}^{+}$non-Tregs) and only with $\mathrm{CD}^{+}$non-Tregs ( $n=6$ /group). (G) Significant decrease of BM Tregs in the IFNAR1-KO group (IFNAR1-KO Tregs + $\mathrm{CD}^{+}$nonTregs) compared with wild-type group ( $n=3 /$ group) by FACS. (H) Representative FACS analysis of BM Tregs of mice transferred with IFNAR1-KO Tregs compared with mice transferred with wild-type Tregs. (I) FACS analysis of checkpoint-related molecules on BM Tregs obtained from mice injected with IFNAR1KO Tregs and wild-type Tregs. Significant decrease of LAC3 was observed in BM Tregs obtained from mice injected with IFNAR1-KO mice ( $n=3$ /group). $P$ value determined by 2 -tailed $t$ test. Error bars indicate SD. ulated expression of IFNA2, one of the INF- $\alpha$ homologous genes (38), and IRF3 (39) contributed to core enrichment score in this data set. Further analysis showed significantly increased levels of IRF3 and other members of the IFN regulatory factors, including IRF4, IRF5, and IRF9 (Figure 6D). Similarly, there was an increase in the expression of IFNA1 and IFNA2 isoforms, and another key component of the IFN induction pathway, MAVS protein $(40,41)$, in MM samples, compared with healthy controls. Interestingly, lower expression of TLR2 and TLR8 was also observed in MM patients, pointing to concurrent deregulation of TLR signaling in malignant plasma cells (Figure 6D).
We next sought to determine the prognostic role of genes regulating type 1 IFN in MM, and therefore analyzed the survival probability of these genes in a large data set of newly diagnosed MM patients ( $n=542$, GSE2658). Median modus was used for cutoff determination, $P$ values were corrected for multiple testing, and R2 visualization platform (http://r2.amc.nl) was used for calculation. We found a significant decrease in the overall survival of patients with high levels of IFNA1 (Figure 6E), supporting the results of our murine experiments and indicating that type 1 IFN is indeed an essential player in MM progression. Interestingly, lower levels of endosomal TLR8 (42) were also associated with poor out- 
come in MM patients (Figure 6F), further suggesting a high impact for deregulated type 1 IFN pathways on MM progression.

Blocking IFNAR1 suppresses inhibitory function of Tregs and inhibits myeloma progression. In order to validate the effect of myelomacell-secreted type 1 IFN on Treg function, we cocultured Vk* MYC cells with sorted Tregs and treated them with either anti-IFNAR1 antibody, which blocks ligand-induced intracellular signaling and induction of type I IFN-induced biologic responses (43), or isotype control. Treg proliferation, analyzed by Ki-67 expression, was significantly decreased in the IFNAR1-treated group, compared with control. This led to a decrease in Treg expansion in IFNAR1-treated cells, compared with control (Figure 7A-D). This study was also performed with 5TGM1 MM cells, which were capable of inducing expansion of C57BL/KaLwRij Tregs in a transwell coculture assay (Supplemental Figure 7B). Once more, IFNAR1-blocking antibody treatment led to decreased Treg proliferation and expansion, compared with treatment with isotype control (Supplemental Figure 7C). Collectively, our data demonstrate that myeloma-cellsecreted type 1 IFN induces proliferation and expansion of Tregs when present in the myeloma microenvironment.

Lastly, we sought to validate in vivo the significance of IFNAR1 as a critical regulator of Treg function and expansion in MM. For this, we performed a $\mathrm{T}$ cell transfer experiment using IFNAR1-KO Tregs; we injected sorted wild-type (C57BL/6) $\mathrm{CD}^{+} \mathrm{CD} 25^{-}$nonTregs, wild-type Tregs, or IFNAR1-KO Tregs into RAG2-KO mice, followed by $\mathrm{Vk}^{*} \mathrm{MYC}$ transplantation, and then analyzed tumor growth and survival (Figure 7E). Mice injected with IFNAR1-KO Tregs lacked the effect of wild-type Tregs in enhancing progression of MM, with survival rates similar to those of mice that had been injected with non-Tregs ( $\mathrm{CD}^{+}$only) (Figure $7 \mathrm{~F}$ ). Namely, Tregs lacking IFNAR1 failed to suppress MM-directed $\mathrm{T}$ cell immunity, and that failure was further reflected in their reduced proliferation and expansion in vivo. While an increase in the number of Tregs was observed in mice that received wild-type Tregs, in accordance with the results of our previous experiments, the expansion was abrogated in the IFNAR1-KO mice, confirming that MM cells induce Treg expansion through type 1 IFN signaling (Figure 7G). As expected, the BM Tregs of mice transferred with IFNAR1-KO Tregs had lower expression of IFNAR1, compared with mice transferred with wild-type Tregs (Figure 7H). Moreover, when comparing BM Tregs between mice injected with wild-type Tregs and those injected with IFNAR1-KO Tregs, we observed a significant decrease in the expression of LAG3 in the latter group (Figure 7I), indicating that type 1 IFN signaling is also partly responsible for checkpoint molecule expression on Tregs in the tumor microenvironment. Thus, our data demonstrate that blocking IFNAR1 reduces myeloma-mediated Treg immunosuppression, leading to better survival rates of myeloma-injected animals.

\section{Discussion}

Increased presence of Tregs in the tumor microenvironment of cancer patients has been associated with both improved $(44,45)$ and poor prognosis $(46,47)$. A few studies described an increase in the number of Tregs in the PB of patients with MM, compared with healthy donors $(10,12,13,48)$, indicating a potential role for Tregs in MM in particular. However, the mechanisms of Treg expansion in $\mathrm{MM}$, as well as the role of Tregs in MM progression, have not been fully elucidated (49). Understanding those mechanisms is critical, as it could be key in developing new immune therapies in MM. In the present study, we identified a feedback loop between myeloma cells and Tregs, wherein type 1 IFN secreted from MM cells induces immunosuppression caused by Tregs, thus promoting MM progression. Interrupting this loop by blocking IFNAR1 signaling relieves immunosuppression and prevents/delays tumor progression.

First, we confirmed that Tregs increase in number and expand in the BM with disease progression, a phenomenon that was detectable in the $\mathrm{BM}$ at an earlier time point, compared with the $\mathrm{PB}$, indicating that myeloma-promoting Treg deregulation first occurs in the context of the tumor microenvironment. In addition, we showed that myeloma-associated BM Tregs highly express immune checkpoint receptor molecules, like PD1, LAG3, and TIM3. Again, in contrast to Teffs, where these molecules are markers of exhaustion, in Tregs, they signify enhanced immunosuppressive function (7). Thus, our data indicate that Tregs in the myeloma microenvironment are activated not only in terms of quantity, but also quality.

Next, we sought to determine the importance of Tregs in myeloma progression. Our in vivo Treg depletion and Treg transfer experiments demonstrated a strong impact of Tregs on the progression of MM, as continuous Treg depletion in vivo led to tumor remission and prolonged survival of $\mathrm{Vk}^{*} \mathrm{MYC}$-injected mice. These results are consistent with other tumor models where enhancement of antitumor immune responses occurs with depletion of Tregs by targeting highly expressed surface receptors $(17,50-52)$.

Interestingly, we found that the cellular content of the $\mathrm{BM}$ after Treg depletion reflects an environment hostile for tumor growth, with a significant increase in the Teff/Treg ratio as well as $\mathrm{B}$ cells and macrophages, indicating that these cells could be critical for the reconstitution of the immune microenvironment and the prevention of tumor progression in MM.

To further explore mechanisms by which myeloma-associated Tregs are activated, we then conducted RNA-seq, comparing BM Tregs of $\mathrm{Vk}^{*} \mathrm{MYC}$-injected mice to BM Tregs of control mice. In addition to checkpoint-related genes and Treg effector genes being significantly upregulated, the type 1 IFN pathway was found to be highly enriched in the gene signature of myeloma-associated Tregs. Validating these results, expression data from MM patients also demonstrated upregulation of well-known genes regulating type 1 IFN induction pathways, such as IRF3, IRF4, IRF5, IRF9, and MAVS protein in malignant plasma cells; these genes were associated with worse prognosis in the survival of MM patients, underscoring the impact of IFNA1 on survival outcome.

Type 1 IFNs are known players in a variety of diseases, such as viral infection, inflammatory bowel disease, systemic lupus erythematosus, and type I diabetes (53), but their role in myeloma remains poorly characterized. Recently, it was shown that type 1 IFN signaling is essential for maintenance of FOXP3 expression, as well as Treg suppressive function under inflammatory conditions (54), while IFNAR signaling was shown to promote Treg development and function under stress conditions (55). In the cancer setting, IFNARmediated signaling was found to be necessary for tumor-infiltrating Treg activation and production of IL-10, as well as suppression of T helper 17 cells, in the tumor microenvironment of colon adenocarcinoma (56). In our system, we demonstrated that type 1 IFN is critical for Treg function using in vitro and in vivo models. 
Based on this, we thus hypothesized that suppression of the type 1 IFN pathway could inhibit myeloma-mediated Treg expansion and hinder tumor progression. Indeed, anti-IFNAR1 antibody led to the suppression of the proliferation of myeloma-associated Tregs in an in vitro coculture assay, as well as extending the survival of myeloma-injected mice in a $\mathrm{T}$ cell transfer experiment with IFNAR1-KO Tregs.

In conclusion, we have unprecedented findings demonstrating that Treg function and homeostasis is regulated by type 1 IFN secreted from myeloma cells in the BM microenvironment, effectively evoking a feedback loop between Tregs and myeloma cells that promotes disease progression and leads to inferior outcome. Prolonged survival in MM mouse models was achieved by Treg depletion and by blocking IFNAR1 signaling and suppressing myeloma-mediated Treg induction and proliferation. To the best of our knowledge, this is the first report indicating that type 1 IFN signaling is a potentially novel therapeutic target for regulating Treg-mediated immunosuppression in MM.

\section{Methods}

Cells. The 5TGM1 cell line was a gift from David G. Roodman (Indiana University, Indianapolis, Indiana, USA). The $\mathrm{Vk}^{*} \mathrm{MYC}$-derived transplantable Vk12598 cell line (18) was provided by Marta Chesi and P. Leif Bergsagel and was maintained and expanded by in vivo retransplantation in Rag2-KO mice, similar to previous reports $(5,57)$. For in vitro experiments, these cell lines were cultured in RPMI- 1640 containing 10\% FBS (Sigma-Aldrich), $2 \mathrm{mM} \mathrm{L-glutamine,} 100 \mathrm{U} / \mathrm{ml}$ penicillin, and $100 \mu \mathrm{g} / \mathrm{ml}$ streptomycin (GIBCO). Human MM1S cells were purchased from ATCC. The human OPM2 myeloma cell line was purchased from DMSZ.

$\mathrm{PB}$ cells or BM samples from patient/healthy donor aspirates were collected at the Dana-Farber Cancer Institute. The CD138-negative BM cell fraction was isolated using MACS technology (Miltenyi Biotec).

Mice. C57BL/6, DEREG, Rag2-KO, and IFNAR1-KO mice, all under the C57BL/6 background, were purchased from Jackson Laboratories. C57BL/KaLwRij mice were purchased from Harlan Laboratories.

Vk12598 cells $\left(2 \times 10^{6}\right)$ were injected intravenously (i.v.) into each mouse for T cell analysis or survival studies. 5 TGM1 cells $\left(3 \times 10^{6}\right)$ were injected i.v. into C57BL/KaLwRij mice for $\mathrm{T}$ cell analysis. BM cells and $\mathrm{PB}$ from mice were harvested for CyTOF analysis by BM aspiration from the femur and by submandibular bleeding, respectively. For other experiments, BM cells were obtained through flushing of femurs with $1 \times$ PBS, as previously described (58).

Treg depletion study. Treg depletion was performed during Vk*MYC progression on DEREG mice by injecting intraperitoneally $10 \mathrm{ng} / \mathrm{g} /$ mouse of DT on day 5, 12, and 19 after Vk* MYC injection. For control groups, the same amount of DT or PBS was injected into C57BL/6 or DEREG mice, respectively. Depletion of Tregs using anti-CD25 antibody was performed as described in previous reports $(250 \mu \mathrm{g}$ of antimurine $\mathrm{CD} 25 \mathrm{mAb}$ ) (27). Isotype antibody and anti-CD25 antibody treatment was performed on day -4 and day 10 after tumor injection.

$T$ cell transfer study. Tregs were obtained from spleens of C57BL/6 or IFNAR1-KO mice by flow-sorting $\mathrm{CD} 4^{+} \mathrm{CD} 25^{+}$cells using a FACSAria II flow cytometry system (BD Biosciences). Similarly, $\mathrm{CD}^{+}{ }^{+}$nonTregs were obtained from C57BL/ 6 splenocytes by sorting $\mathrm{CD}^{+}{ }^{+} \mathrm{CD} 25$ cells. Tregs $\left(1 \times 10^{6}\right)$ and non-Tregs $\left(3 \times 10^{6}\right)$ were injected i.v. into Rag2-KO mice, followed by Vk12598 cell injection 5 days afterwards.
Analysis of cells by CyTOF. Sample staining and data analysis were performed as reported previously (57). Briefly, cells were first stained with MaxPar Intercalator-Rh (Fluidigm Sciences Inc.) for determination of viable cells. After blocking with mouse Fc Receptor Blocking Reagent (Miltenyi Biotec), cell surface marker antibodies were added and stained. Following staining, cells were washed with MaxPar Cell staining buffer (Fluidigm Sciences Inc.) and fixed/permeabilized with Fix/Perm buffer (PBS with 1.6\% paraformaldehyde and $0.3 \%$ saponin). Cells were then washed with cell staining buffer containing $0.3 \%$ saponin, followed by intracellular antibody staining. For mass cytometric analysis, cells were stained with MaxPar Ir DNA intercalator (Fluidigm Sciences Inc.) overnight at $4^{\circ} \mathrm{C}$. Cells were then washed with cell staining buffer and then finally with MaxPar water (Fluidigm Sciences Inc.) alone. Cells were acquired on a CyTOF2 mass cytometer (Fluidigm Sciences Inc.). The acquired data were analyzed in Cytobank (https://www. cytobank.org/). The gating strategy for Tregs is described in Supplemental Figure 1A.

The CD138-negative populations of SMM patients or healthy donors' BM cells were thawed, washed, and barcoded using the CellID 20-Plex Pd Barcoding Kit (Fluidigm Sciences Inc.). After barcoding, cells were incubated with human surface marker antibodies as a single multiplexed sample (TIGIT-143Nd, CD152 [CTLA4]-152Sm, PD1-175Lu, TIM3-153Eu [Maxpar], and Maxpar Human T-Cell Phenotyping Panel Kit, 16 Marker). The cells were fixed with $4 \%$ paraformaldehyde, permeabilized with ice-cold methanol, and incubated with antibodies against intracellular proteins (FOXP3 [clone PCH101], custom conjugation with $165 \mathrm{Ho}$ by Harvard Medical Area CyTOF Antibody Resource and Core, Boston, Massachusetts, USA). Then the cells were washed, resuspended at $1 \times 10^{6}$ cells $/ \mathrm{ml}$, and acquired on a CyTOF Helios mass cytometer (Fluidigm Sciences Inc.). Downstream analysis of the individual component samples was performed after running the debarcoding application. Human T cell populations were gated according to the Maxpar Human T Cell Phenotyping Panel Kit.

Flow cytometric analysis. Anti-mouse antibodies against CD3 (clone 17A2), CD4 (clone GK1.5), CD8 (clone 53-5.8), PD1 (clone 29F.1A12) (Biolegend); CD25 (clone PC61.5), LAG3 (clone C9B7W), TIM3 (clone 8B.2C12), B220 (clone RA3-6B2), FOXP3 (clone FJK16s), Ki-67 (clone SolA15) (eBioscience); CD138 (clone 281-2) (BD Biosciences); and anti-human antibodies against CD4 (RPA-T4), FOXP3 (PCH101) (eBioscience); and CD25 (BC96, Biolegend) were used for the FACS analyses. Cells were surface stained, fixed/permeabilized, and stained intracellularly using the FOXP3/Transcription Factor Staining Buffer Set (eBioscience) according to the manufacturer's protocol. The data were acquired using a FACSCanto II flow cytometer (BD Biosciences) and were analyzed in Cytobank.

RNA-seq analysis. Total RNA was isolated from flow-sorted $\mathrm{CD}^{+}$FOXP3-EGFP ${ }^{+}$cells acquired from the BM of $\mathrm{Vk}^{*} \mathrm{MYC}$-injected and control mice using an RNeasy Micro kit (QIAGEN). Whole RNA was subjected to library preparation using a NEBNext Ultra RNA Library prep for Illumina kit (New England Biolabs). Quality control of the libraries was evaluated by Bioanalyzer analysis with High Sensitivity chips (Agilent Technologies). Sequencing was performed on a HiSeq 2000 (Illumina) by $2 \times 50$ bp paired-end reads at the Biopolymers Facility of Harvard Medical School. We used Picard and STAR to process and align the RNA-seq reads with a mouse reference genome ( $\mathrm{mm10}$ ) and to compute a series of quality-control metrics. RNA-seq transcript abundances were 
estimated using RSEM (59). Genes with low expression (RSEM expected counts) were filtered out and differential gene expression between different samples was determined using DESeq2 (60). Differential expression was selected based on fold changes greater than 2.0 and a $P$-value threshold of 0.05. Sequencing data were deposited in the NCBI's Gene Expression Omnibus database (GEO GSE109533). GSEA was used to identify significantly enriched pathways (61), with false discovery rate (FDR) less than 0.25 and $P$ less than 0.05. Gene sets were downloaded from the Broad Institute's MSigDB (http://www.broadinstitute.org/ gsea/index.jsp). The human data were deposited in the NCBI's GEO database (GSE6477 and GSE2658).

ELISA. Murine myeloma cell lines (Vk12598 cells and 5TGM1 cells) and (C57BL/6 or C57BL/KaLwRij) B cells were seeded into 12 -well plates at a concentration of $2 \times 10^{5}$ cells $/ \mathrm{ml}$ and cultured for 24 hours. Murine IFN- $\alpha$ and IFN- $\beta$ levels in the cell culture supernatants were detected using VeriKine-HS Mouse IFN ELISA Kits (PBL Assay Science) according to the manufacturer's protocol.

Treg induction and expansion assay. $\mathrm{CD}^{+} \mathrm{CD} 25^{-}$non-Tregs (induction assay) at $2 \times 10^{5}$ or $\mathrm{CD} 4{ }^{+} \mathrm{CD} 25^{+}$Tregs (expansion assay), isolated from C57BL/ 6 or C57BL/KaLwRij splenocytes were cocultured with 1 $\times 10^{5}$ MM1S or OPM2 cells in the presence of recombinant murine IL-2 (1,000 IU/ml; R\&D Systems), anti-CD3 antibody ( $5 \mu \mathrm{g} / \mathrm{ml}$; eBioscience, clone 145-2C11), and anti-CD28 antibody ( $5 \mu \mathrm{g} / \mathrm{ml}$; eBioscience, clone 37.51 ) for 72 hours. TGF- $\beta$ (10 ng/ml; R\&D Systems) was added for the induction assay. Anti-IFNAR1 antibody (Biolegend) or isotype control antibody were added to the appropriate wells at $100 \mu \mathrm{g} / \mathrm{ml}$.

Human $\mathrm{CD}^{+} \mathrm{CD} 25^{-}$non-Tregs or CD19-expressing B cells were obtained from fresh $\mathrm{PB}$ cells using $\mathrm{CD} 4{ }^{+} \mathrm{CD} 25^{+}$Regulatory T Cell Isolation Kit or CD19 Microbeads (Miltenyi Biotec). Briefly, red blood cells were lysed (RBC lysis buffer, Biolegend), washed, and isolated according to the manufacturer's instructions. The $\mathrm{CD} 4^{+} \mathrm{CD} 25^{-}$non-Tregs $(2 \times$ $10^{5}$ ) were cocultured with $1 \times 10^{5} \mathrm{MM} 1 \mathrm{~S}$ or OPM2 cells in the presence of recombinant human IL-2 (1,000 IU/ml; R\&D Systems) and Treg Suppression Inspector beads (Miltenyi Biotec) at a 1:1 bead/cell ratio. Transwell permeable inserts with a pore size of $0.4 \mu \mathrm{m}$ were used to prevent cell-cell interactions (Corning).
Statistics. One-way ANOVA was used when 3 or more independent groups were compared. The unpaired Student's $t$ test was used to compare 2 independent groups with Bonferroni's correction for multiple comparisons. For survival data, Kaplan-Meier curves were plotted and compared using a log-rank test. All tests were 2-tailed. A P value less than 0.05 was considered statistically significant. Analysis was performed with GraphPad Prism software.

Study approval. All mice were treated, monitored, and sacrificed in accordance with an approved protocol of the Dana-Farber Cancer Institute Animal Care and Use Committee.

Human studies were approved by the Dana-Farber Cancer Institute Institutional Review Board. Written informed consent was obtained from all patients and healthy volunteers in accordance with the Declaration of Helsinki protocol.

\section{Author contributions}

YK, OZ, JA, and IMG conceived and designed the study. YK, OZ, MM, KK, THM, NM, M. Reidy, YJS, M. Rahmat, MB, PL, ESK, S. Tsukamoto, SM, JS, S. Takagi, YM, DH, and AS acquired data. YK, OZ, MM, JP, SM, JS, YTT, MC, PLB, AMR, JA, and IMG analyzed and interpreted data. YK, OZ, JP, RSP, AMR, JA, and IMG wrote, reviewed, and/or revised the manuscript.

\section{Acknowledgments}

Funding support was provided by NIH grants R01 CA18168301A1 and R01 AI134842-01 as well by the Leukemia and Lymphoma Society (LLS SCOR, 7004-14).

Address correspondence to: Irene M. Ghobrial, Department of Medical Oncology, Dana-Farber Cancer Institute, Harvard Medical School, 450 Brookline Avenue, Boston, Massachusetts 02115, USA. Phone: 617.632.4198; Email: irene_ghobrial@dfci.harvard. edu. Or to: Jamil Azzi, Transplantation Research Center, Brigham and Women's Hospital, Harvard Medical School, 221 Longwood Avenue, Boston, Massachusetts 02115, USA. Phone: 617.525.7359; Email: jazzi@rics.bwh.harvard.edu.
1. Topalian SL, Drake CG, Pardoll DM. Immune checkpoint blockade: a common denominator approach to cancer therapy. Cancer Cell. 2015;27(4):450-461.

2. Armand P. Immune checkpoint blockade in hematologic malignancies. Blood. 2015;125(22):3393-3400.

3. Ansell SM, et al. PD-1 blockade with nivolumab in relapsed or refractory Hodgkin's lymphoma. N Engl J Med. 2015;372(4):311-319.

4. Landgren O. Monoclonal gammopathy of undetermined significance and smoldering multiple myeloma: biological insights and early treatment strategies. Hematology Am Soc Hematol Educ Program. 2013;2013:478-487.

5. Guillerey C, et al. Immunosurveillance and therapy of multiple myeloma are CD226 dependent. J Clin Invest. 2015;125(5):2077-2089.

6. Suen H, Brown R, Yang S, Ho PJ, Gibson J, Joshua D. The failure of immune checkpoint blockade in multiple myeloma with PD-1 inhibitors in a phase 1 study. Leukemia. 2015;29(7):1621-1622.

7. Pardoll D, Drake C. Immunotherapy earns its spot in the ranks of cancer therapy. J Exp Med. 2012;209(2):201-209.

8. Fontenot JD, Gavin MA, Rudensky AY. Foxp3 programs the development and function of $\mathrm{CD} 4^{+} \mathrm{CD} 25^{+}$regulatory T cells. Nat Immunol. 2003;4(4):330-336.

9. Penaloza-MacMaster P, et al. Interplay between regulatory $\mathrm{T}$ cells and $\mathrm{PD}-1$ in modulating $\mathrm{T}$ cell exhaustion and viral control during chronic LCMV infection. JExp Med. 2014;211(9):1905-1918.

10. Beyer $M$, et al. In vivo peripheral expansion of naive $\mathrm{CD}^{+} \mathrm{CD} 25^{\text {high }} \mathrm{FoxP}^{+}$regulatory $\mathrm{T}$ cells in patients with multiple myeloma. Blood. 2006;107(10):3940-3949.

11. Prabhala RH, et al. Dysfunctional T regulatory cells in multiple myeloma. Blood. 2006;107(1):301-304.

12. Feyler S, et al. CD4(+)CD25(+)FoxP3(+) regulatory T cells are increased whilst CD3(+)CD4(-)CD8(-) alphabetaTCR $(+)$ double negative $\mathrm{T}$ cells are decreased in the peripheral blood of patients with multiple myeloma which correlates with disease burden. Br J Haematol. 2009;144(5):686-695.
13. Muthu Raja KR, Rihova L, Zahradova L, Klincova M, Penka M, Hajek R. Increased T regulatory cells are associated with adverse clinical features and predict progression in multiple myeloma. PLoS ONE. 2012;7(10):e47077.

14. Bryant $\mathrm{C}$, et al. Long-term survival in multiple myeloma is associated with a distinct immunological profile, which includes proliferative cytotoxic T-cell clones and a favourable Treg/Th17 balance. Blood Cancer J. 2013;3:e148.

15. Glatman Zaretsky A, et al. T regulatory cells support plasma cell populations in the bone marrow. Cell Rep. 2017;18(8):1906-1916.

16. Feyler S, et al. Tumour cell generation of inducible regulatory T-cells in multiple myeloma is contact-dependent and antigen-presenting cellindependent. PLoS One. 2012;7(5):e35981.

17. Feng X, et al. Targeting CD38 suppresses induction and function of $\mathrm{T}$ regulatory cells to mitigate immunosuppression in multiple myeloma. Clin Cancer Res. 2017;23(15):4290-4300.

18. Chesi M, et al. Drug response in a genetically engineered mouse model of multiple 
myeloma is predictive of clinical efficacy. Blood. 2012;120(2):376-385.

19. Chesi M, et al. AID-dependent activation of a MYC transgene induces multiple myeloma in a conditional mouse model of post-germinal center malignancies. Cancer Cell. 2008;13(2):167-180.

20. Bendall SC, et al. Single-cell mass cytometry of differential immune and drug responses across a human hematopoietic continuum. Science. 2011;332(6030):687-696.

21. Azzi J, et al. The novel therapeutic effect of phosphoinositide 3-kinase- $\gamma$ inhibitor AS605240 in autoimmune diabetes. Diabetes. 2012;61(6):1509-1518.

22. Wang Y, et al. Rig- $\mathrm{I}^{-/}$mice develop colitis associated with downregulation of $\mathrm{G}$ alpha i2. Cell Res. 2007;17(10):858-868.

23. Braga WM, et al. FOXP3 and CTLA4 overexpression in multiple myeloma bone marrow as a sign of accumulation of $\mathrm{CD} 4(+) \mathrm{T}$ regulatory cells. Cancer Immunol Immunother. 2014;63(11):1189-1197.

24. Sharma P, Allison JP. The future of immune checkpoint therapy. Science. 2015;348(6230):56-61.

25. Camisaschi C, et al. LAG-3 expression defines a subset of CD4(+)CD25(high)Foxp3(+) regulatory $\mathrm{T}$ cells that are expanded at tumor sites. J Immunol. 2010;184(11):6545-6551.

26. Lahl K, et al. Selective depletion of Foxp $3^{+}$regulatory $\mathrm{T}$ cells induces a scurfy-like disease. J Exp Med. 2007;204(1):57-63.

27. Grauer OM, et al. Elimination of regulatory $\mathrm{T}$ cells is essential for an effective vaccination with tumor lysate-pulsed dendritic cells in a murine glioma model. Int J Cancer. 2008;122(8):1794-1802.

28. Takeda I, et al. Distinct roles for the OX40-OX40 ligand interaction in regulatory and nonregulatory T cells. J Immunol. 2004;172(6):3580-3589.

29. Valzasina B, Guiducci C, Dislich H, Killeen N, Weinberg AD, Colombo MP. Triggering of OX 40 (CD134) on CD4(+)CD25+ T cells blocks their inhibitory activity: a novel regulatory role for OX40 and its comparison with GITR. Blood. 2005;105(7):2845-2851.

30. Shevach EM. Mechanisms of foxp $3^{+}$T regulatory cell-mediated suppression. Immunity. 2009;30(5):636-645.

31. Zheng Y, et al. Regulatory T-cell suppressor program co-opts transcription factor IRF4 to control $\mathrm{T}(\mathrm{H}) 2$ responses. Nature. 2009;458(7236):351-356.

32. Chaudhry A, et al. $\mathrm{CD} 4^{+}$regulatory $\mathrm{T}$ cells control TH17 responses in a Stat3-dependent manner. Science. 2009;326(5955):986-991.

33. Koch MA, Tucker-Heard G, Perdue NR, Killebrew JR, Urdahl KB, Campbell DJ. The transcription factor T-bet controls regulatory $\mathrm{T}$ cell homeostasis and function during type 1 inflam- mation. Nat Immunol. 2009;10(6):595-602.

34. Schneider MA, Meingassner JG, Lipp M, Moore $\mathrm{HD}$, Rot A. CCR7 is required for the in vivo function of $\mathrm{CD} 4^{+} \mathrm{CD} 25^{+}$regulatory T cells. J Exp Med. 2007;204(4):735-745.

35. Yamazaki T, et al. CCR6 regulates the migration of inflammatory and regulatory T cells. J Immunol. 2008;181(12):8391-8401.

36. Lunardi S, Lim SY, Muschel RJ, Brunner TB. IP-10/CXCL10 attracts regulatory T cells: Implication for pancreatic cancer. Oncoimmunology. 2015;4(9):e1027473.

37. Redjimi N, et al. CXCR3 ${ }^{+}$T regulatory cells selectively accumulate in human ovarian carcinomas to limit type I immunity. Cancer Res. 2012;72(17):4351-4360.

38. Hillyer P, et al. Expression profiles of human interferon-alpha and interferon-lambda subtypes are ligand- and cell-dependent. Immunol Cell Biol. 2012;90(8):774-783.

39. Génin P, Vaccaro A, Civas A. The role of differential expression of human interferon--a genes in antiviral immunity. Cytokine Growth Factor Rev. 2009;20(4):283-295.

40. Kawai T, et al. IPS-1, an adaptor triggering RIG-Iand Mda5-mediated type I interferon induction. Nat Immunol. 2005;6(10):981-988.

41. Seth RB, Sun L, Ea CK, Chen ZJ. Identification and characterization of MAVS, a mitochondrial antiviral signaling protein that activates NFkappaB and IRF 3. Cell. 2005;122(5):669-682.

42. Iwasaki A, Medzhitov R. Toll-like receptor control of the adaptive immune responses. Nat Immunol. 2004;5(10):987-995.

43. Sheehan KC, et al. Blocking monoclonal antibodies specific for mouse IFN-alpha/beta receptor subunit 1 (IFNAR-1) from mice immunized by in vivo hydrodynamic transfection. J Interferon Cytokine Res. 2006;26(11):804-819.

44. Tzankov A, Meier C, Hirschmann P, Went P, Pileri SA, Dirnhofer S. Correlation of high numbers of intratumoral $\mathrm{FOXP}^{+}$regulatory $\mathrm{T}$ cells with improved survival in germinal center-like diffuse large B-cell lymphoma, follicular lymphoma and classical Hodgkin's lymphoma. Haematologica. 2008;93(2):193-200.

45. Farinha P, Al-Tourah A, Gill K, Klasa R, Connors JM, Gascoyne RD. The architectural pattern of FOXP3-positive T cells in follicular lymphoma is an independent predictor of survival and histologic transformation. Blood.2010;115(2):289-295.

46. Curiel TJ, et al. Specific recruitment of regulatory $\mathrm{T}$ cells in ovarian carcinoma fosters immune privilege and predicts reduced survival. Nat Med. 2004;10(9):942-949.

47. Bates GJ, et al. Quantification of regulatory T cells enables the identification of high-risk breast cancer patients and those at risk of late relapse. JClin Oncol. 2006;24(34):5373-5380.

48. Brimnes MK, et al. Increased level of both $\mathrm{CD}^{+}{ }^{+} \mathrm{FOXP}^{+}$regulatory $\mathrm{T}$ cells and $\mathrm{CD} 14^{+} \mathrm{HLA}-$ $\mathrm{DR}^{-/ \text {low }}$ myeloid-derived suppressor cells and decreased level of dendritic cells in patients with multiple myeloma. Scand J Immunol. 2010;72(6):540-547.

49. Kawano Y, et al. Targeting the bone marrow microenvironment in multiple myeloma. Immunol Rev. 2015;263(1):160-172.

50. Marabelle A, et al. Depleting tumor-specific Tregs at a single site eradicates disseminated tumors. J Clin Invest. 2013;123(6):2447-2463.

51. Bulliard Y, Jolicoeur R, Zhang J, Dranoff G, Wilson NS, Brogdon JL. OX40 engagement depletes intratumoral Tregs via activating Fc $\gamma \mathrm{Rs}$, leading to antitumor efficacy. Immunol Cell Biol. 2014;92(6):475-480.

52. Klages K, et al. Selective depletion of Foxp3 ${ }^{+}$ regulatory $\mathrm{T}$ cells improves effective therapeutic vaccination against established melanoma. Cancer Res. 2010;70(20):7788-7799.

53. González-Navajas JM, Lee J, David M, Raz E. Immunomodulatory functions of type I interferons. Nat Rev Immunol. 2012;12(2):125-135.

54. Lee SE, et al. Type I interferons maintain Foxp3 expression and T-regulatory cell functions under inflammatory conditions in mice. Gastroenterology. 2012;143(1):145-154.

55. Metidji A, Rieder SA, Glass DD, Cremer I, Punkosdy GA, Shevach EM. IFN- $\alpha / \beta$ receptor signaling promotes regulatory $\mathrm{T}$ cell development and function under stress conditions. JImmunol. 2015;194(9):4265-4276.

56. Stewart CA, et al. Interferon-dependent IL-10 production by Tregs limits tumor Th17 inflammation. J Clin Invest. 2013;123(11):4859-4874.

57. Moschetta M, et al. Targeting vasculogenesis to prevent progression in multiple myeloma. Leukemia. 2016;30(5):1103-1115.

58. Roccaro AM, et al. C1013G/CXCR4 acts as a driver mutation of tumor progression and modulator of drug resistance in lymphoplasmacytic lymphoma. Blood. 2014;123(26):4120-4131.

59. Li B, Dewey CN. RSEM: accurate transcript quantification from RNA-Seq data with or without a reference genome. BMC Bioinformatics. 2011;12:323.

60. Love MI, Huber W, Anders S. Moderated estimation of fold change and dispersion for RNA-seq data with DESeq2. Genome Biol. 2014;15(12):550.

61. Subramanian A, et al. Gene set enrichment analysis: a knowledge-based approach for interpreting genome-wide expression profiles. Proc Natl Acad Sci U S A. 2005;102(43):15545-15550. 